\title{
Detection of indeterminacies in corrected ECG signals using parameterized multidimensional independent component analysis
}

\author{
M.P.S. Chawla* \\ Department of Electrical Engineering, Indian Institute of Technology, Roorkee 247667, India
}

( Received 9 January 2008; final version received 9 May 2008)

\begin{abstract}
Independent component analysis (ICA) is a new technique suitable for separating independent components from electrocardiogram (ECG) complex signals. The basic idea of using multidimensional independent component analysis (MICA) is to find stable higher dimensional source signal subspaces and to decompose each rotation into elementary rotations within all two-dimensional planes spanned by the coordinate axes useful for diagnostic information of heart. In this paper, ability of ICA for parameterization of ECG signals was felt to reduce the amount of redundant ECG data. This work aims at finding an independent subspace analysis (ISA) model for ECG analysis that allows applicability to any random vectors available in an ECG data set. For the common standards for electrocardiography (CSE) based ECG data sets, joint approximate diagonalization of eigen matrices (Jade) algorithm is used to find smaller subspaces. The extracted independent components are further cleaned by statistical measures. In this study, it is also observed that the value of kurtosis coefficients for the independent components, which represents the noise component, can be further reduced using parameterized multidimensional ICA (PMICA) technique. The indeterminacies if available in the ECG data are to be analysed also using modified version of Jade algorithm to PMICA and parameterized standard ICA (PsICA) for comparative studies. The indeterminacies if available in the ECG data are reduced in PMICA better in comparison to the analysis done using PsICA. The simulation results obtained indicate that ICA definitely improves signal-noise ratio (SNR) like the other higher order digital filtering methods like Kalman, Butterworth etc. with minimum reconstruction errors. Here, it is also confirmed that re-parameterization of the standard ICA model results into a 'component model' using MICA technique, which is geometric in spirit and free of indeterminacies existing in sICA model.
\end{abstract}

Keywords: electrocardiogram; parameterization; multidimensional independent component analysis; indeterminacies; reconstruction; statistical thresholds

\section{Introduction}

Principal component analysis (PCA) is used to reduce dimensionality and for feature extraction of the electrocardiogram (ECG) data prior to or after performing independent component analysis (ICA). PCA alone is not a very appropriate technique for the visualization of ECG data and non-linear dimensionality reduction algorithms from the ECG morphological point of view $[24,28]$. This is due to the fact that it can only uncover linear relationships in the ECG data, and can be designed to find directions in the ECG data with the highest variance that may not be always the most informative directions. PCA

\footnotetext{
*Email: mpschawla@rediffmail.com; mpschawla@gmail.com
} 
algorithm will always provide a projection to an orthogonal basis whether the signal of interest can be best decomposed or interpreted within this basis or not [27,28].

ICA has been widely used in data analysis and decomposition in the last three decades and typically aims to solve the blind source separation problem in which a set of unknown sources are mixed in some way to form the data which is available $[39,66]$. The increasing demand of ICA is generally applied to the ECG data, following the generative ICA model in order to guarantee a feasible algorithm to attain valid results on morphological basis [60].

In the literature, the terms blind source separation and independent component analysis are often used indifferently: they refer to the same model (1) with the same assumptions, pursue the same objectives and are addressed with the same algorithms [41,51]. It is also unfortunate because the term 'analysis' refers to the idea of decomposition into smaller, simpler elements and very often this decomposition is into a sum of terms, calling for an additive model rather than a multiplicative model $[1,20]$. Uniqueness and separability results play a central role in solving blind source separation (BSS) problems since they allow algorithms to apply ICA in order to uniquely (except for scaling and permutation) find the original mixing matrices $[4,6,16,30,33,34,43,44,59,64,83]$.

In ECG, electroencephalogram (EEG) or magnetoencephalogram (MEG) signal processing, large set of electrodes (from 10 in ECG to more than 100 in EEG and MEG) are used, and the signals recorded using electrodes are related to the electric or magnetic fields due to the electrical activity of heart or neurons [53]. ECG source separation consists in retrieving unknown ECG signals, which are observed through unknown mixture of these [53].

ICA has been successfully applied to the processing of electroencephalography signals. Various methods available in the literature have been proposed in the identification of components related to artefacts in both EEG and MEG. In brain research, ICA has previously been applied in a multitude of cases, including EEG, MEG and magnetic resonance imaging (MRI) analysis [18,38,49,77]. ICA has already proven to be a valuable tool for detecting and enhancing relevant source subspace brain signals, while suppressing irrelevant noise and artefacts such as those produced by muscle activity and eye blinks [6,50,51].

ICA opens new and useful windows into phenomena contained in multi-channel ECG records by separating ECG data recorded at multiple electrodes into a sum of independent components $[21-23,28,42,56]$. In particular, ICA appears to be a generally applicable and effective method for removing a wide variety of artefacts and noise from ECG records [25,26,28,42,62]. Basically ECG processing consists of three main steps:

(i) A measurement, which produces some raw ECG data

(ii) A data analysis algorithm that extracts parameters of interest from the ECG data and (iii) A decision or interpretation rule based on the extracted parameters.

Modelling ECG data as consisting of convolutive as well as static independent processes allows a richer palette for source modelling, possibly leading to more complete signal independence. ICA yields independent components (ICs) displaying more clearly the investigated properties of the original ECG sources [28,41]. The goal of multidimensional independent component analysis (MICA) for ECG analysis lies in the linear separation of ECG data into statistically independent groups of ECG signals [72,75].

Independent subspace analysis (ISA), nowadays an attractive alternative for dimensionality reduction, however, are currently limited by the assumption of equal group sizes or less general semi-parametric models. By introducing the concept of irreducible independent subspaces or components, one can present a generalization to a parameter free mixture of an ECG model. However, the condition of utmost one Gaussian 
can be relaxed by including previous results on non Gaussian component analysis of an ECG data set. After introduction of this general model, joint block diagonalization with unknown block sizes can be discussed, on the basis of a simple extension to joint approximate diagonalization of eigen matrices (Jade) can be algorithmically performed to better understand the subspace analysis. Simulations of different typical case studies carried out on an ECG data set only can confirm the feasibility of the developed ISA algorithm [75].

\subsection{Existing ECG techniques: A review}

During ECG recording some of the unwanted signals (artefacts) interfere with ECG signal, as a result of which analysis of the ECG signal and extraction of diagnostic features from ECG becomes difficult [20,42,76]. Filtering the noise components instead of discarding them may result in a processed signal, which is more representative of the original data $[12,23,28,81]$. It should be noted that the ECG does not have the same appearance in all the leads of the standard 12-lead system used in clinical practice. The ECG polarity and the shape of the ECG constituent waves may change depending on the lead used and its location $[24,41,80]$.

Recently, there are many approaches which have been proposed in literature that involve techniques for computer processing of 12-lead ECG in order to diagnose certain diseases. Numerous techniques $[8,9,12,14,41,50,76,78,81]$ have been developed to recognize and analyse ECG waves, ranging from digital filtering techniques to intelligent techniques such as neural networks, fuzzy logic and spectro-temporal-based techniques. A first group of methods to interpret the ECG significance uses a morphological analysis [23,28,42]. A second group of techniques for computer analysis of ECG uses statistical models $[7,20,37,57,63]$. A third category of methods corresponding to neural models becomes a powerful concurrent to statistical ones for ECG signal classification [9,24,81]. In the past, a systematic comparison among several neural paradigms and architectures and between neural and statistical approach has been performed on a large validated ECG databases, in order to investigate the behaviour and the features of each approach in the particular problem of the ECG analysis. In the wavelet or discrete Fourier transform (DFT) domain approaches, it is common practice to window and taper the ECG data; hence, the choice of the optimal window size is a problem in practice $[8,9,78]$.

When applying unsupervised learning techniques in ECG data analysis, a key question is whether the estimated parameters of the studied system are reliable. In other words, can one assess the quality of the result produced by the proposed learning technique? In the past years, several ECG analysers based on statistical methods, clustering methods, expert systems and Markov models have been developed and implemented to solve the problem of the time consuming ECG analysis [1,12,20,81]. That their reliability is less, together with their high sensitivity to noise and their failure to deal with new or ambiguous patterns, leads the research towards investigation of new analysis techniques. Artificial neural networks (ANN) have been often proposed as good classifiers when non-linear separation borders are required and incomplete or ambiguous input ECG patterns can be found. In the last few years the connectionist approach has been applied to the ECG analysis with promising results $[22,23,27]$. Once the reliability of a solution can be estimated, then one can use the information for model/algorithm selection purposes, for testing model validity and for improving the used algorithm [25,61]. Note that re-sampling is completely general and can be applied to assess reliability of any unsupervised learning algorithms, e.g. projection techniques such as instance independent component analysis, principal component analysis, kernel PCA, clustering and so on [5,11,36,51]. 


\subsection{PCA preprocessing}

Signal processing in general has tremendously changed during the last 20 years and it is expected to change even more in the years to come. What was earlier visualized as digital signal processing now forms only a small part of the new concept of signal processing which might be more adequately explained as the methods of analysing, manipulating and conveying natural information? Feature extraction is basically reduction of the available information maintaining ECG morphology $[28,29,61]$. Features are representatives of identification to a particular subject or specimen. Analysis and feature extraction from electrocardiograms is difficult until and unless artefacts and noise from the ECG are removed, there are so many techniques available in the literature [25,26,41].

PCA is the optimal linear technique, which retains the maximum amount of variance (amongst all linear projections) within the projected feature space $[28,36,40]$. PCA is often used in ECG pattern recognition and the transform produce features that are mutually uncorrelated. The results obtained by the Karhunen-Loeve Transform (KLT) solution are optimal, when dimensionality reduction is the goal and it is desired to minimize the approximation mean square error. PCA preprocessing process consists of centring as well as whitening steps and the centring step is done by subtracting the mean of the observed ECG data, which provides zero mean ECG data. The whitening step is used to remove the correlation between the observed ECG data. A common method to achieve whitening is by the eigenvalue decomposition of the covariance matrix of the mixed ECG signal [13,28,37]. Figure 1(a) shows the basic ECG waveform, representing its basic component waves, whereas Figure 1(b) explains a philosophy for PCA preprocessing of ECG data. Some preprocessing $[28,57]$ is useful before attempting to estimate $\mathbf{w}$. The important points to be considered are:

(i) The observed signals should be centred by subtracting their mean value $\mathrm{E}\{\mathbf{x}\}$.

(ii) Then they are whitened, which means they are linearly transformed so that the components are uncorrelated and has unit variance.

(iii) Whitening can be performed via eigen value decomposition of the covariance matrix, $\mathbf{V} \mathbf{\Lambda} \mathbf{V}^{\mathbf{T}}$, where $\mathbf{V}$ is the matrix of orthogonal eigenvectors and $\mathbf{\Lambda}$ is a diagonal matrix with the corresponding eigenvalues. The whitening is done by multiplication with the transformation matrix $\mathbf{P}$.

$$
\begin{gathered}
\mathbf{P}=\mathbf{V} \boldsymbol{\Lambda}^{-1 / 2} \mathbf{V}^{\mathrm{T}} \\
\mathbf{z}=\mathbf{P}\{\mathbf{x}-\mathrm{E}[\mathbf{x}]\} .
\end{gathered}
$$

The covariance of the whitened data $\mathrm{E}\left[\mathbf{Z Z}^{\mathrm{T}}\right]$ equals the identity matrix, I. For simplicity, let $\mathbf{x}$ be the centred mixed vector, $\{\mathbf{x}-\mathrm{E}[\mathbf{x}]\}$ i.e. $\mathbf{x}=\{\mathbf{x}-\mathrm{E}[\mathbf{x}]\}$.
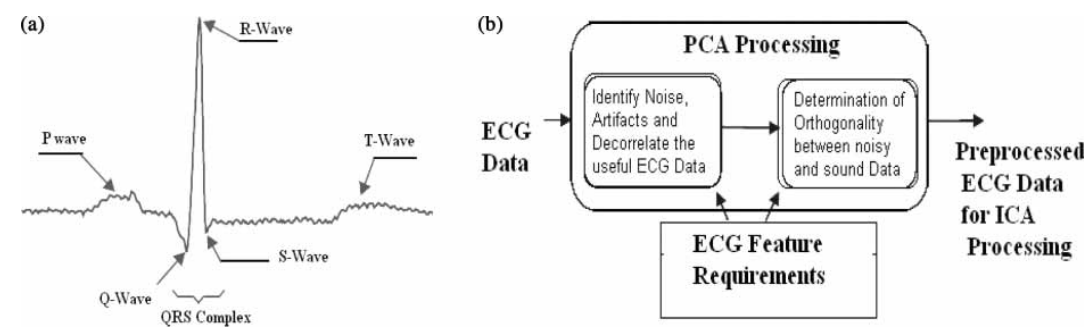

Figure 1. (a) ECG waveform. (b) PCA preprocessing of ECG data. 


\subsection{Blind source separation}

Source separation methods have been developed exclusively for a long time for linear mixtures, instantaneous as well as convolutive, and more recently by a few researchers even for nonlinear mixtures in biomedical applications [55]. In most of the general cases, the underlying assumptions made on the sources are that they are statistically independent. The ICA or BSS model can be uniquely identified provided it is properly parameterized in terms of one-dimensional subspaces. From this standpoint, BSS/ICA model performs better if generalized to multidimensional components $[18,35,37,40,68]$. In BSS methods, the ECG electrodes are assumed to measure a mixture of some latent sources, and both the ECG sources and the mixing process are unknown. Some undesired signals may be superimposed on ECG signals of interest and must be considered artefacts. These artefacts may have a biological origin, like reciprocal contaminations of muscle activity and heart cycles, respectively in ECG and EMG recordings $[8,9,11,50]$. These motion artefacts produce base line drifts, which can compromise vital signs parameters extrapolation $[22,23,27-29,42]$. In ECG recordings, it is possible that artefacts and noise can cause the loss of the main features, like QRS complex, $\mathrm{P}$ and $\mathrm{T}$ waves.

Linear as well as non-linear filtering and adaptive filtering can be listed among the techniques available, in order to recover the features of interest from ECG recordings. Other approaches take advantage from the multi-channel nature of the acquisitions with the aim of searching for common features present in different ECG channels acquired simultaneously. Such approaches may be useful in situations when the frequency content of signal and noise overlap, and filtering operation cannot reduce noise without losing signal diagnostic information.

Second order blind identification (SOBI) techniques consist of separating an ECG mixture of independent sources with different spectral content through second order analysis, considering also temporal information of the ECG sources. For this purpose, SOBI methods aim to find a transformation that diagonalizes the cross correlation matrix at several lags simultaneously. Since there may exist no transformation which accomplishes that condition, a function that measures the diagonalization at different lags must be defined in order to maximize the joint diagonalization criteria. From Darmois's result [17], one deduces that this problem has no solution for mutually independent Gaussian sources, with temporally independent and identically distributed (iid) samples. Then, since the Gaussian iid model has no solution, one must add priors, which are threefold $[53,54]$ for success of an application:

(i) Non Gaussian iid.

(ii) Gaussian but not temporally independent (the first i of iid is relaxed), i.e. temporally correlated.

(iii) Gaussian, but not identically distributed (id of iid is relaxed), i.e. non stationary sources.

\subsection{Standard ICA (sICA)}

Independence is not always a strong constraint of ICA and independence assumption is generally true, but the nature of signals suggests using other priors: for instance, ECG is sparse signal, and most of the biological signals are temporally correlated and nonstationary [53]. ICA can be viewed as a maximum projection algorithm so as to find the sources that are maximally independent. This is quite meaningful when studying cardiac processes, as it is unreasonable to assume that the activity in one part of the heart is completely independent of the activity in various other parts of the heart [66]. Strictly 
speaking, it is not necessary to assume for the information maximization in ICA that the sources do not have a Gaussian distribution. In the case of Gaussian distributed signals $[22,28,31,39,66,67]$, ICA merely degenerates to regular old PCA with summarized variance equivalent to information and uncorrelated components also being independent.

ICA is a statistical technique for obtaining independent sources, $\mathbf{s}$ from their linear mixtures, $\mathbf{x}$ when neither the original sources nor the actual mixing matrix, $\mathbf{A}$ are known as given by Equation (3). This model is easily applied by exploiting higher order signal statistics and optimization techniques. For the application of ICA, it is assumed that the ECG signal, $\mathbf{x}(\mathbf{t})$ is generated from sources $\mathbf{s}(\mathbf{t})$ with a linear mixing procedure

$$
\mathbf{x}(\mathbf{t})=\mathbf{A} \mathbf{s}(\mathbf{t}),
$$

where, ' $\mathbf{t}$ ' is a vector of the sampling time with length $T$.

Also, $\mathbf{x}(\mathbf{t})=\left[\mathbf{x}_{1}(\mathbf{t}) \ldots \mathbf{x}_{n}(\mathbf{t})\right]$ is $\mathbf{n} \times \mathrm{T}$ matrix of noise reduced ECG signal recorded from ' $n$ ' sensors. $\mathbf{s}(\mathbf{t})=\left[\mathbf{s}_{1}(\mathbf{t}) \ldots \mathbf{s}_{m}(\mathbf{t})\right]$ is a $m \times$ T matrix of $m$ sources activations.

$\mathbf{A}$ is an unknown $n \times m$ full rank mixing matrix [66].

Consider the noiseless complex linear instantaneous blind source separation model with as many sources as sensors: $\mathbf{x}=\mathbf{A s}$. Here $\mathbf{s}$ is an independent complex-valued ' $\mathbf{n}$ ' dimensional random vector and $\mathbf{A}$ is an invertible complex matrix [6]. The task of linear BSS is to find $\mathbf{A}$ and $\mathbf{s}$ given only $\mathbf{x}$. For the sake of simplicity, the discussion is restricted throughout to the case of zero-mean real signals. The source separation problem may be stated as: Identify mixing matrix $\mathbf{A}$ and/or estimate the source signals based only on observations of $\mathbf{x}$ and assuming only

(i) Statistical independence of the 'sources' and

(ii) Linear independence of the columns of $\mathbf{A}$.

The strength of this model is that the two independence assumptions stated above are physically plausible in several instances and are strong enough to provide some kind of identifiability, thus alleviating the need of any further modelling of the source distributions or of the mixing matrix $[28,42,46,51,66]$. ICA aims to find the demixing matrix $\mathbf{w}$, such that

$$
\mathbf{s}=\mathbf{w} \mathbf{x}
$$

where $\mathbf{w}$ is whitening matrix.

The mixing/demixing process can be described as a change of coordinates. This means we consider the sources $\mathbf{S}_{i}$ as components of a vector $\mathbf{s}$ with respect to an orthonormal basis and the observations $\mathbf{x}_{j}$ as the components of the very same vector in terms of a different basis. The demixing matrix thus helps to find the sources $\mathbf{s}$. To simplify the estimation of these independent sources we can start by decorrelating the mixtures, (whitening or sphering). This makes the covariance matrix of $\mathbf{x}$ diagonal and its components of unit variance. ICA then uses higher order statistical information (kurtosis, negentropy, etc) to estimate the independent sources $[11,20,42]$. The prime requirement for applying ICA is that a number of simultaneously measured ECG signals carry linear combinations of the original source ECG signals.

\subsection{Outline of the present analysis}

In the present work, Jade algorithm for sICA is to be first applied to 3-channel ECG, common standards for electrocardiography (CSE) database files and the ECG waveforms are separated as independent components. The statistical corrections are then applied to 
the extracted independent components to find noise or artefact components. In this paper, it is also proposed that the ability of ICA for parameterization of ECG signals may be necessary at times $[22,29,61]$. Properly parameterized ECG signals provide a better view to the extracted ECG signals, while reducing the amount of ECG data [11,70]. ICA yields independent components displaying more clearly the investigated properties of the original ECG sources. ICs of properly parameterized ECG signals may also be more readily interpretable than the measurements themselves, or their ICs. The basic idea of using MICA $[15,20]$ is to find stable higher dimensional source signal subspaces and to decompose each rotation into elementary rotations within all two-dimensional planes spanned by the coordinate axes for useful diagnostic information of the heart. In this work, multidimensional blind source separation (MBSS) [75] i.e. the recovery of underlying sources $\mathbf{s}$ from an observed mixture $\mathbf{x}$ of ECG signals is utilized. As usual, $\mathbf{s}$ has to fulfil additional properties such as independence or diagonality of the auto-covariances (if $\mathbf{s}$ possesses time structure). However in contrast to ordinary blind source separation, MBSS is more general as some source ECG signals are allowed to possess common statistics. One possible solution for MBSS is multidimensional independent component analysis.

The idea of MICA is that one does not require the full independence of the transform $\mathbf{y}=\mathbf{w x}$ but only mutual independence of certain tuples, $y_{i 1}, \ldots, y_{i 2}$. If the size of all tuples is restricted to one, this reduces to ordinary ICA. In general, of course the tuples could have different sizes, but for the sake of simplicity we assume that they all have the same length $k[6,15,73,74]$.

In this study, it is also to be verified that the value of kurtosis and Varvar coefficients for the independent components, which represents the noise and artefacts components, can be further reduced using parameterized multidimensional ICA (PMICA) technique or not. The indeterminacies if available in the ECG data are to be analysed also using a modified version of Jade algorithm to PMICA and parameterized standard ICA (PsICA) for comparative studies.

The salient objectives of the proposed work are:

(a) This study aims at finding an ISA model for ECG analysis that allows applicability to either one or all random vectors available in an ECG data set.

(b) For the CSE based ECG data sets, it is desired to apply joint approximate diagonalization of eigen matrices (Jade) algorithm and its modified version are suggested as well re-implemented in this study, for estimating the smaller subspaces.

(c) The basic idea of applying MICA in this study, was to find stable higher dimensional source signal subspaces and to decompose each rotation into elementary rotations within all two-dimensional planes spanned by the coordinate axes for getting useful diagnostic information of heart for CSE based ECG data sets with and without noise as well as artefacts.

(d) Parameterization of ECG signals is to be properly done, in order to provide a better view to the extracted ECG signals, while reducing the amount of ECG data, which may be of redundant nature, possessing almost no useful diagnostic information.

\section{Summarization of issues and imposed conditions before applying ICA}

Because of the very weak assumptions in applying this, the problem is often referred to as BSS. The method based on the property of source independence has been called ICA [52,55]. In fact, one often has priors on the ECG signals, but not necessary always. A natural idea is then to add the priors in the proposed or developed ECG model, for simplifying or improving the separation methods [55]. Nonlinear mixtures are not in 
general separable $[69,82]$ and a practically important case of nonlinear mixtures is post nonlinear (PNL) mixtures [55], in which a linear mixture is followed by nonlinear sensors. It has been shown that PNL mixtures are separable using statistical independence, too $[2,55,69,82]$, with the same indeterminacies as linear mixtures. However, if some weak prior information about the source signals is available, then the performance of the source separation algorithms may be significantly improved. Thus, these methods are not 'blind' but 'semi-blind' [55].

The answers to the above raised issues and summarization of the facts that, what are the advantages of applying ICA in ECG analysis is: [23,25,26,28,29]

(i) ICA is a form of BSS.

(ii) It can solve and handle time delay with ambiguity problems if available in the ECG data.

(iii) ICA assumes the ECG sources as linear mixtures.

(iv) ICA allows physicians an alternative higher order statistical technique for better ECG interpretation.

(v) It performs better in terms of yielding a cleaned ECG signal as good as higher order digital filters.

(vi) ICA is better at recovering specific points on the ECG such as the $R$-peak, RR interval which is necessary for obtaining the heart rate.

\subsection{Why are extensions to ICA required?}

Independent component analysis is a multidimensional signal processing technique to separate signals from different sources into distinct components. Once separated, components classified as noise may be discarded and the remaining components used to reconstruct the pure signal. However, due to the imperfect nature of the ICA technique, substantial data may be lost, when the 'noise' components were removed or discarded $[15,22,23,29,41,42]$.

An important structural aspect in the search for decompositions is to have the knowledge of the number of possible solutions for a problem, as well to find the number of indeterminacies involved in solving a problem [60]. The knowledge of this is essential, without which the result of any ICA or ISA algorithm cannot be compared with other possible and available solutions, as well the BSS would not be possible. Clearly, given an ISA solution, invertible transforms in each component (scaling matrices $\mathbf{L}$ ) as well as permutations of components of the same dimension (permutation matrices $\mathbf{P}$ ) give again an ISA of $\mathbf{X}$. In some of the special cases of ICA, scaling and permutation are already in all indeterminacies given that at most one Gaussian is contained in $\mathbf{X}$ [65]. This is one of the important points of ICA, allowing its usage for solving BSS problems and hence stimulating many biomedical applications. It has been proved by some of the researchers that, also for $k$-ISA, scalings and permutations are nothing but the indeterminacies [71] with some additional restrictions imposed on the model and are not the weak restrictions to the model [75]. Blind identification of multi-channel ECGs based on second-order statistics (SOS) is unique only to within multiplication by a unitary matrix, whereas with higher-order statistics, the multiplication ambiguity is reduced to the class of signed permutation matrices. Of course, higher-order statistics (HOS) do not offer a panacea for all problems and one may have to use statistics and tools other than SOS and HOS.

ICA methods using time structure are better suited to biosignal analysis than HOS methods as ICA can solve and handle time delay with ambiguity problems if available in 
the ECG data. In addition, ICA with time structure is better at recovering specific points on the ECG such as the $R$-peak, RR interval which is necessary for obtaining the heart rate. On the other hand, conventional ICA algorithms when applied to the signals like ECG do not consider the data asymmetry and time structure, as a result of which the extracted ICs may not correspond to the intuitively expected directions.

\subsection{Conditions of existence for ICA solutions}

From a statistical point of view, since the problem has no solution for Gaussian iid signals, three types of statistical priors are possible: sources are non Gaussian iid, sources are Gaussian temporally correlated, and sources are Gaussian non-stationary [55]. Initially, even if it was not clearly stated [53], the problem has been related to the non Gaussian iid model, and has been referred to as BSS. The non Gaussian property appears clearly in the Comon's theorem [32] for linear mixtures. Consider, $\mathbf{x}=\mathbf{A s}$, is a $p$-dimension regular mixture of mutually independent random variables, with at most one Gaussian, $\mathbf{y}=\mathbf{B x}$ has mutually independent components if $\mathbf{B A}=\mathbf{P D}$, where $\mathbf{P}$ and $\mathbf{D}$ are permutation and diagonal matrices, respectively [53]. This theorem is only based on the independence of random variables and the independence criterion involves either explicitly or implicitly higher order statistics (at least more than two), but does not take into account the order of the ECG samples. It means that the iid assumption is not required, which is just a default assumption. Consequently, ICA methods can be applied for iid as well as for non iid sources, but it does not work successfully for Gaussian sources [52,55]. Moreover, the theorem points out that in reference to ECG analysis, the ECG sources cannot be exactly estimated, but only up to a scale and a permutation. These are the typical indeterminacies of source separation in the analysis when dealing with linear mixtures $[17,32,55]$.

Of course, without other assumptions, the problem of ECG processing cannot be solved directly. Basically, it is necessary in any ECG analysis to have certain conditions such as [55]:

(i) Priors or information about the nature of the ECG mixtures should be known.

(ii) It is very important to choose an appropriate separating transform $\mathbf{B}$ suitable for the mixture transform $\mathbf{A}$.

(iii) The sources as well as the source properties, even if weak, is necessary for determination of the separating transform $\mathbf{B}$.

\subsection{Indeterminacies of complex ICA}

The main interest of source separation problem is its relevance in many application domains, providing the maximum information is more than the multidimensional observations [53]. In the simplest case, this diversity is spatial and is obtained by using many sensors. Thus, for providing efficient solutions, as is required for any estimation problem, one has to choose carefully the following ingredients:

(1) The model of mixture, i.e. what is the relationship between the observations, $\mathbf{x}$ and the sources, $\mathbf{s}$.

(2) The criterion is the source independence relevant or not?

(3) Do the sources have other properties that could be used in temporal coloration, non-stationarity, sparsity, discrete values, etc?

(4) The optimization algorithm [55].

Given a complex $n$-dimensional random vector $\mathbf{x}$, a matrix $\mathbf{w}$ is called complex ICA of $\mathbf{x}$ if $\mathbf{w} \mathbf{x}$ is independent as a complex random vector. It can be proved that $\mathbf{w}$ and $\mathbf{v}$ are complex 
ICA's of $\mathbf{x}$ if and only if $\mathbf{w}^{-1} \sim \mathbf{v}^{-1}$, that is if they differ by right multiplication by a complex scaling and permutation matrix $[6,15]$. This is equivalent to calculating the indeterminacies of the complex BSS model. The task of linear BSS is to find A and $\mathbf{s}$ given only $\mathbf{x}$.

An obvious indeterminacy of this problem is that $\mathbf{A}$ can be found only up to equivalence because of scaling $\mathbf{L}$ and permutation matrix $\mathbf{P}$. Thus, $\mathbf{x}=\mathbf{A} \mathbf{L} \mathbf{P} \mathbf{P}^{-1} \mathbf{L}^{-1} \mathbf{S}$ and $\mathbf{P}^{-1} \mathbf{L}^{-1} \mathbf{s}$ is also independent. Thus, it can be shown that under mild assumptions to $\mathbf{s}$ there are no further indeterminacies of complex BSS. Various algorithms for solving the complex BSS problem have been proposed as discussed in [6]. It can be shown that many cases where complex BSS is applied can in fact be reduced to using real BSS algorithms. This is the case if either the sources or the mixing matrix are real. The latter, for example, occurs after Fourier transformation of signals with time structure. If the sources are real, then the above complex model can be split up into two separate real BSS problems: $\operatorname{Re}(\mathbf{x})=\operatorname{Re}(\mathbf{A}) \mathbf{s}$ and $\operatorname{Im}(\mathbf{x})=\operatorname{Im}(\mathbf{A}) \mathbf{s}$. Solving both of these real BSS equations yields $\mathbf{A}=\operatorname{Re}(\mathbf{A})+i \operatorname{Im}(\mathbf{A})$.

Of course, $\operatorname{Re}(\mathbf{A})$ and $\operatorname{Im}(\mathbf{A})$ can only be found except for scaling and permutation. By comparing the two recovered source random vectors (using for example mutual information of one component of each vector), we can however assume that the permutation and then also the scaling indeterminacy of both recovered matrices is the same, which allows the algorithm to correctly put A back together. Similarly, also separability of this special complex ICA problem can be derived from the well-known separability results in the real case $[6,15]$. If the mixing matrix is known to be real, then again splitting up Equation (3) into real and complex parts yields, $\operatorname{Re}(\mathbf{x})=\mathbf{A} \operatorname{Re}(\mathbf{s})$ and $\operatorname{Im}(\mathbf{x})=\mathbf{A} \operatorname{Im}(\mathbf{s})$. 'A' can be found from either equation. If both real and complex samples are to be used in order to increase precision, those can simply be concatenated in order to generate a twice as large sample set mixed by the same mixing matrix $\mathbf{A}$. In terms of random vectors, this means working in two disjoint copies of the original probability space [6].

\subsection{Mutual information (MI) and non Gaussianity}

Finally, for linear mixtures, one can derive other families of algorithms by considering particular factorizations of the inverse transform, which is a matrix G. Due to the scale indeterminacy, one can look for unit variance source and a usual idea is to factorize $\mathbf{G}=\mathbf{U w}$, where $\mathbf{w}$ is a whitening matrix and $\mathbf{U}$ is an orthogonal matrix. After estimating $\mathbf{w}$ such that, $\mathrm{E}\left[(\mathbf{w} \mathbf{x})(\mathbf{w x})^{\mathrm{T}}\right]=\mathbf{I}$ with second order statistics, one can estimate $\mathbf{U}$ by minimizing MI with higher order statistics [53].

\section{Multidimensional ICA (MICA)}

Multidimensional ICA [6,72] has first been introduced by Cardoso [15] using geometrical motivations. HyvParinen and Hoyer then presented a special case of multidimensional ICA which they called independent subspace analysis [74]; there the dependence within a $k$-tuple is explicitly modelled enabling one to propose better algorithms without having to resort to the problematic multidimensional density estimation. The crucial idea how to find stable higher dimensional source signal subspaces is to calculate not the overall rotation for each direction, but to decompose each rotation into $\mathbf{N}(\mathbf{N}-1) / 2$ elementary rotations within all two-dimensional planes spanned by the coordinate axes [15]. If the used BSS algorithm is successful in separating the independent subspaces, the separability matrix should have a block structure that groups together one-dimensional ICA projections that belong to the same independent subspace. Thus, a reliable independent subspace should become clearly separated from every other subspace. 
A random vector $\mathbf{Y}$ is called an independent component of the random vector $\mathbf{X}$, if there exists an invertible matrix $\mathbf{A}$, and a decomposition $\mathbf{X}=\mathbf{A}(\mathbf{Y}, \mathbf{Z})$ such that $\mathbf{Y}$ and $\mathbf{Z}$ are stochastically independent. The goal of a general independent subspace analysis or MICA is the decomposition of an arbitrary random vector $\mathbf{X}$ into independent components. If $\mathbf{X}$ is to be decomposed into one-dimensional components, this coincides with ordinary independent component analysis. Similarly, if the independent components are required to be of the same dimension $k$, then this is denoted by multidimensional ICA of fixed group size $k$ or simply $k$-ISA. So 1-ISA is equivalent to ICA [75]. A different extension of ICA is given by topographic ICA [48], where dependencies between all components are assumed. A special case of multidimensional ICA is complex ICA and can be presented where dependence is allowed between real-valued couples of random variables [6]. PCA provides a decomposition of a second-order vector $\mathbf{x}$ as the sum of its projections onto the principal axis of its covariance matrix. The PCA components are geometrically orthogonal by construction and also statistically orthogonal or uncorrelated [24]. The more ambitious ICA approach is to look for components which are not necessarily geometrically orthogonal but are statistically independent (that is 'more than statistically orthogonal' since independence is much stronger than mere un-correlation). Standard ICA algorithms $[23,28,29,42,56]$ that are applied to such a data set will produce one-dimensional source estimates that are as independent as possible, which means they will still find the right decomposition, but they are forced to select as well a decomposition of the actually multidimensional components. Thus standard ICA techniques $[1,6,15,51,73]$ are able to find the multidimensional source signal subspaces, but they choose an (arbitrary) basis within these subspaces. A matrix $\mathbf{w}$ is called a $k$-multidimensional ICA of an $n$ dimensional random vector $\mathbf{x}$ if $\mathbf{w} \mathbf{x}$ is $k$-independent. If $k=1$, this is the same as ordinary ICA. As usual MICA can solve the multidimensional BSS problem $\mathbf{x}=\mathbf{A s}$; where $\mathbf{A}$ and $\mathbf{s}$ is a $k$-independent $n$-dimensional random vector. Finding the indeterminacies of multidimensional ICA then shows that $\mathbf{A}$ can be found except for $k$-equivalence (separability), because if $\mathbf{x}=\mathbf{A s}$ and $\mathbf{w}$ is a demixing matrix [6]. The problem is then to decide which of the one-dimensional source space estimates given by the algorithm should be grouped together. One may think of other ways of decomposing a Gaussian vector into independent components but it does not seem possible to define a decomposition that would be invariant $[37,40]$. In consequence, a special treatment is reserved to Gaussian components: they should not be split into independent subcomponents but rather all the Gaussian components (if any) should be kept pieced together as a unique component. The ICA model (1) can be rewritten as an additive, component-based model:

$$
\mathbf{x}=\sum_{p=1}^{n} \mathbf{x}_{p}
$$

This (admittedly trivial) rewriting of the original model calls for a change of standpoint. While model (3) is a multiplicative model reading: the observed vector is the product of a mixing matrix $\mathbf{A}$ by a source vector $\mathbf{s}$, model (5) is an additive model reading: the observed vector is a sum of ' $n$ ' one-dimensional independent vectors $\mathbf{x}_{1}, \ldots, \mathbf{x}_{n}$. The new view of ICA introduced in Equation (5) above is 'matrix-free'. Here, we discuss how it can be parameterized and why the appropriate parameterization is uniquely determined contrarily to the matrix-based parameterization of model (3). In the component model (5), the smallest subspace containing the $p$ th component is referred to as the "component (sub) space' for the $p$ th component. This is indeed the one-dimensional linear subspace spanned 
by the $p$ th column of $\mathbf{A}$. By focusing on the spaces containing each component rather than on the columns of $\mathbf{A}$, we obtain the desired result of getting rid of the indeterminations of scale and sign. In some sense, we move from an algebraic description of a mixture in terms of a 'mixing matrix' to a geometric description in terms of 'component spaces'. Using MICA, the noiseless linear MBSS problem, $\mathbf{x}=\mathbf{A s}$ can be solved, where the $m n$ dimensional random vector $\mathbf{x}$ is given, and $\mathbf{A}$ and $\mathbf{s}$ are unknown. In the case of MICA, $\mathbf{s}$ is assumed to be $m$-independent $[72,75]$.

It is important to determine the conditions, in order to find firstly how ICA algorithms can be designed to extract one-dimensional components and then to find a suitable criterion for such ICA algorithms, so as to investigate how the algorithms behave, when the ECG processing of a mixture of multidimensional independent components is being carried out. To describe the ECG signals more reliably for better extraction of diagnostic features and morphological understanding, it is intuitively suggested that the existing ICA algorithms can be modelled best with multidimensional components, but has to be carried more rigorously as a future work.

The reasons quoted above are to be carried as 'additional research', since an implicit assumption of common ICA algorithms is that the input signals are symmetric around their mean values, since the mean of the ECG data is initially removed, and the sign of the ICs are not determinable. This means that when applying conventional ICA algorithms to the signals such as ECG without considering the data asymmetry, the extracted ICs do not correspond to the intuitively expected directions for extracting useful morphological features for disease classification.

\subsection{Existing ICA algorithms and limitations}

Signals such as ECG have probability densities that are close to Gaussian, while artefacts such as EEG, EMG, motion artefacts have non Gaussian distributions. ICA has some inherent limitations, due to which identifying the independent component (IC) of interest becomes difficult and highly subjective at times [1,22]. Before giving an interpretation for the extracted ICs, the isoelectric point of the heart needs to be further discussed. The ECG signals are not symmetric around their mean values, and the isoelectric point of the heart slightly differs from the mean values of the data. Meanwhile, an implicit assumption of common ICA algorithms is that the input signals are symmetric around their mean values, since the mean of the data is initially removed, and the sign of the ICs are not determinable. This means that when applying conventional ICA algorithms to signals such as the ECG without considering the data asymmetry, the extracted ICs do not correspond to the intuitively expected directions.

The different existing ICA algorithms [28,57] are Molgedey and Schuster ICA (MSICA), optimized generalized weighted estimator (OGWE), joint approximation diagonalization of eigen matrices (Jade), shifted block blind separation (SHIBBS), kernel-ICA and robust, accurate, direct independent component analysis algorithm (RADICAL) for removal of artefacts and noise from ECG. For assessing the actual independence of the components obtained from ICA/BSS, mutual information is used. MI leads to basic performance tests for any ICA problem and hence different ICA/BSS algorithms can be ranked by how well they perform i.e. whether they find the most independent components.

The limitation of ICA algorithms is that there is no guarantee that any particular algorithm can capture the individual source signals in its components. As discussed above, a number of ICA/BSS based ECG methods have been published till date, but with 
insufficient background to enable the ECG practitioner to choose the best algorithm [28,57]. For the ECG data sets considered here, Jade algorithm is able to find smaller subspaces and can therefore be regarded as the more suitable algorithm in this analysis. By applying Jade algorithm to the orthogonal subspace allows us to extract the other components in the ECG data set and yields the best solution for better feature extraction. The Jade algorithm is more appropriate for separating ECG data, because Jade yields lower matrix entries, i.e. higher reliability. The Jade separability matrix in contrast shows one-dimensional components and two-dimensional components [23,28,29,42,57].

\subsection{Existing approaches of MICA for dependent component analysis}

Generalizations of the ICA model that are required to include the dependencies of multiple one-dimensional components have been studied for a long time [75]. ISA in the terminology of multidimensional ICA has first been introduced by Cardoso [15] utilizing geometrical motivations and his model as well as the related but independently proposed factorization of multivariate function classes [45] is quite general, however no identifiability results applied to an actual data set were shown and discussed, and applicability to an arbitrary random vector was unclear [75]. Later on in Cardoso, in the special case of equal group sizes ( $k$-ISA), uniqueness results were extended from the basic ICA theory [71]. Algorithmic enhancements [75] in this setting have been recently studied by $[65,79]$. Moreover, if the observation contains additional structures such as spatial or temporal structures, these may be used for the multidimensional separation [45,79].

Hyvarinen and Hoyer presented a special case of $k$-ISA by combining it with invariant feature subspace analysis [45]. They model the dependence within a $k$-tuple explicitly and are therefore able to propose more efficient algorithms without having to resort to the problematic multidimensional density estimation. A related relaxation of the ICA assumption is given by topographic ICA [47], where dependencies between all components are assumed and modelled along a topographic structure (e.g. a two-dimensional grid). Bach and Jordan [10] formulate ISA as a component clustering problem, which necessitates a model for inter-cluster independence and intra-cluster dependence [75].

\section{Methodology of corrections applied to extracted ICs}

The idea of ICA is to recover the original signals by assuming that they are statistically independent. $\mathbf{y}$ is independent and it is desired to find how $\mathbf{w}$ maximizes the independence of $\mathbf{y}$. To estimate one of the independent components (ICs), a linear combination of the $x_{i}$ is considered. Let us denote this by,

$$
y=\mathbf{w}^{\mathrm{T}} x=\sum_{i=1}^{n} \mathbf{w}_{i} x_{i},
$$

where the column vector $\mathbf{w}$ is to be determined.

The independent components are determined by applying a linear transformation to the whitened data.

A given component can be obtained using the linear transformation

$$
\mathbf{i}_{\mathbf{c}}=\mathbf{b}_{\mathbf{i}}^{\mathrm{T}} \mathbf{x},
$$

where $\mathbf{i}_{\mathbf{c}}$ gives independent components which is an estimate of the original signal. $\mathbf{b}$ is an appropriate vector to reconstruct the independent components $[1,50,76]$. The ICA 
algorithmic principle is

$$
\begin{gathered}
\mathbf{y}=\operatorname{Est}(\mathbf{s})=\mathbf{w}_{\mathrm{ICA}} \mathbf{x} \\
\mathbf{y}=\mathbf{w} \mathbf{x} .
\end{gathered}
$$

After estimating $\mathbf{A}, \mathbf{w}=\mathbf{A}^{-1}$ is computed and hence

$$
\mathbf{s}=\mathbf{w} \mathbf{x}=\mathbf{A}^{-1} \mathbf{x} .
$$

Further,

$$
\mathbf{s}^{\prime}(\mathbf{t})=\mathbf{w} \mathbf{x}(\mathbf{t}),
$$

where, $\mathbf{s}^{\prime}(\mathbf{t})=\left[\mathbf{s}_{\mathbf{l}}{ }^{\prime}(\mathbf{t}) \ldots \mathbf{s}_{m}{ }^{\prime}(\mathbf{t})\right]$ is $m$-dimensional matrix of the independent components, which represents the estimation of the sources $\mathbf{s}(\mathbf{t})$ and $\mathbf{w}$ is a demixing matrix [66]. Also,

$$
\mathbf{w}=\mathbf{A}^{\prime}+,
$$

where $\mathbf{A}^{\prime+}$ denotes an estimate of the pseudo-inverse of the mixing matrix $\mathbf{A}$. After the identification of artefacts and noise, the ECG signals are reconstructed with the components which have not been labelled as artefacts and noise. Thus, in the remixing matrix, the weight vectors of the identified artefactual and noise ICs have been set to zero, such that

$$
\mathbf{x}^{\prime}(\mathbf{t})=\mathbf{w}_{\text {clean }}^{+} \mathbf{s}^{\prime}(\mathbf{t}),
$$

where $\mathbf{x}^{\prime}(\mathbf{t})$ is the reconstructed artefacts and noise free ECG signal for $n$ sensors, $\mathbf{w}^{+}$is the pseudo-inverse of the demixing matrix $\mathbf{w}$, and $\mathbf{w}_{\text {clean }}^{+}$is the $n \times m$ remixing matrix with its columns corresponding to the artefactual and noise ICs set to zero, $\mathbf{s}^{\prime}(\mathbf{t})$ is the activation matrix of ICs as mentioned above $[22,23,28,29,42,66]$. Using ICA, it is required to derive a 'clean ECG signal' from the source ECG signal to find the content of noise and compare the proposed model with the existing methods and algorithms [3,5,6,11,15,36,58].

Independent component analysis method is proposed where additional knowledge about the time and statistical structure of the ECG sources is incorporated [23,29,42]. ICA yields equations describing the behaviour of the various ECG segments as a function of cardiac cycle time. ICA can be used to synthesize an ECG signal which is a realistic reproduction of the original signal, as well can control parameters such as QRS complex amplitude, rise-time, fall-time and the relative amplitudes of the P \& T-waves [22,28]. The duration of each component will automatically track the selected heart rate in a non-linear fashion, reflecting its true behaviour. It has been reported in the literature that the ICA source estimates promote some inverse process, acting on the ECG observations. In a standard ICA model, this inverse function is a generalized linear function, i.e. a function of the pseudo-inverse of the mixing matrix, the so called un-mixing matrix and the inferred noise model $[8,19,23,28,29,42]$. The intrinsic indeterminacy of scales and signs is a wellknown limitation of the ICA problem as defined in (1). By using ICA, we are estimating the heart sources and the mixing matrix corresponding to the body volume conductor at the same time. However, due to the non-homogeneity of the body volume conductor the original heart signals are not attenuated with the same amount in all directions.

\section{ICA parameterization}

Based on trivial properties of convolution, some common signal parameters also fulfil the ICA mixing model, given that the original ECG measured signals comply with it. By ECG 
signal parameterization $[1,22,70]$, it is meant that the construction of a new ECG signal is possible from any local or global properties, i.e. parameters of the original ECG signal. These properties may be related to a priori known features of the ECG sources. Even with proper ECG parameterizations, it may sometimes be hard or impossible to make an ICA algorithm converge, because of missing or otherwise bad ECG data due to, for example, bad electrode contacts. It is also otherwise possible that the ICA does not converge, or that several runs of ICA are needed. $R$-wave reflects the intensity and direction of progressing ventricular heart muscle depolarization, i.e. contraction. At times, it is desired that the independent components of the parameterization of ECG signals [70] is to be done that would display diagnostic information carried by the parameters more clearly than what is observable from the original ECG measurements or their ICs. It is to be noted that in order for the ICA mixing model (1) to be valid, the parameters have to be derived from the ECG signal amplitudes. ECG parameters describing time durations, e.g. the time periods between consecutive $R$ waves, do not comply with the ICA mixing model. In some cases, the appropriate ECG parameterization [22] may be such that it greatly decreases the amount of ECG data, thus lowering the computational burden on the subsequent ECG analysis. As readily stated by the mixing model (1), each and every measured sample is a linear combination of the samples of the source ECG signals at the same time. Therefore, a set of ECG signals, in which each signal consists of samples from the corresponding original ECG measurement at the time points 's', i.e.

$$
\mathbf{y}^{\prime}=\mathbf{y}(\mathbf{s})
$$

Equation (14) also satisfies the ICA mixing model of (1). Also, it can be shown that ECG signals constructed from time averages of the original measurement ECG samples, or signals resulting from FIR filtering, fulfil the ICA signal model, and may thus be subjected to ICA. However, it is to be noted that even if the ICA algorithm converged and produced ICs, averaging will make the components more Gaussian. Thus, one must pay special attention to the proper interpretability of the components, and avoid lengthy averaging windows $[49,77]$.

In this paper, it is proposed that the ability of ICA for parameterization of ECG signals is required at times. As appropriate ECG signal parameters reveal desired signal properties, ICs of thus parameterized ECG signals may as well display the desired aspects of the question at hand much more clearly than the ICs of the original ECG signals, while possibly also reducing the amount of ECG data to a fraction of the original ECG signal. The proposed ICA algorithm estimates the independence of the original signal and the optimization based on the estimation searches an optimum-restoring matrix. In the

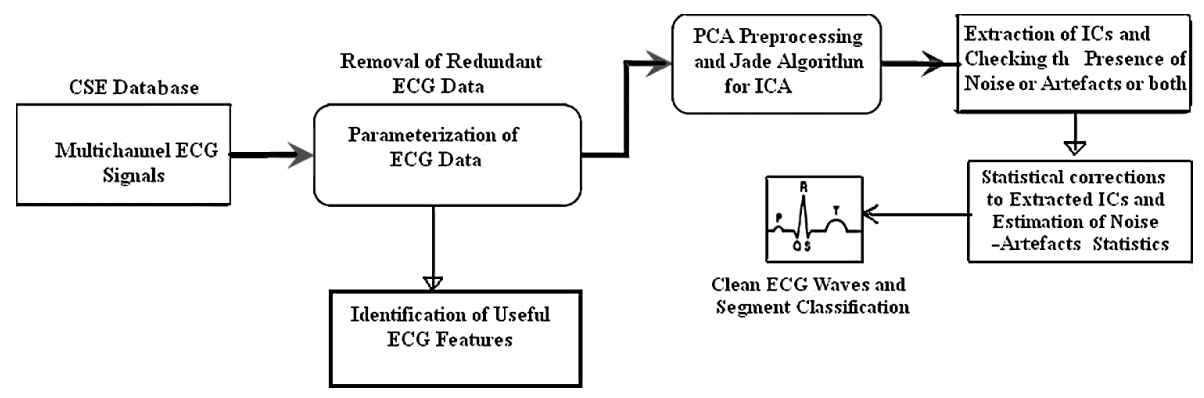

Figure 2. ICA parameterization scheme for ECG segment identification. 


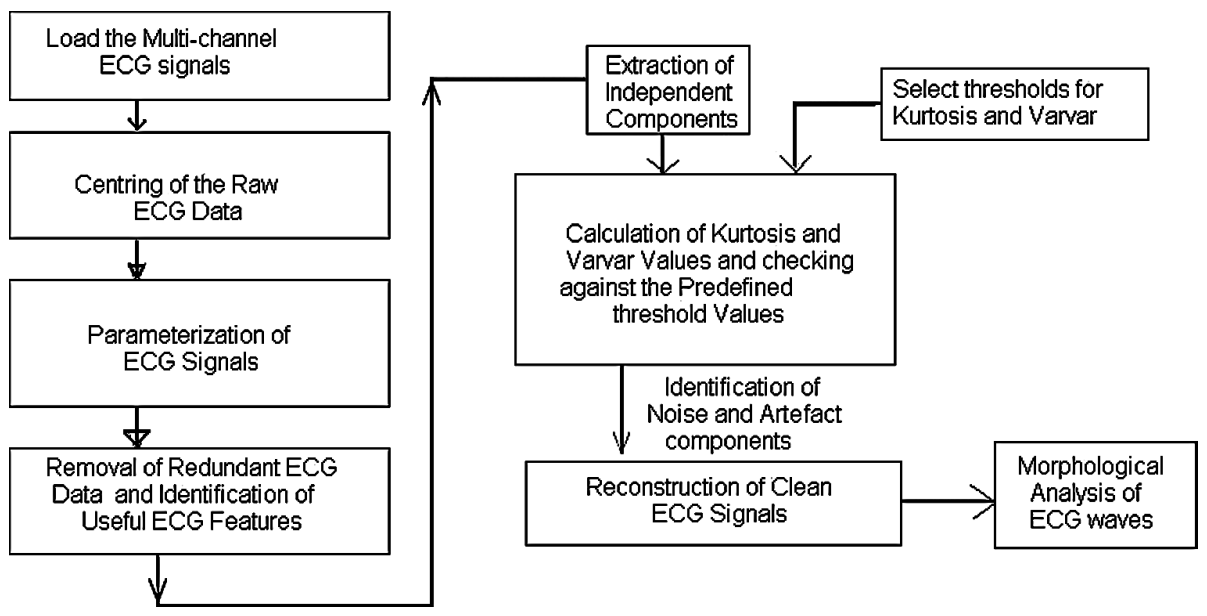

Figure 3. Flow chart of parameterized ICA for morphological analysis of ECG.

proposed model, estimated results are in good agreement with physiological view. The new scheme also makes no assumptions about the non-stationarity of the components, a key assumption in several successful ICA methods, whose relevance to ECG is still unclear $[21,25,26,36]$. Figure 2 depicts ICA parameterization scheme for ECG segment identification and classification. The flow chart of parameterized ICA steps for morphological analysis of ECG waves is depicted in Figure 3.

In this analysis, an efficient approach for artefacts/noise identification with parameterization technique is discussed. The automatic removal of artefacts and noise in ECG signals based on correction procedures adapted to extracted ICs as discussed above, based on statistical thresholds including estimations of the noise statistical properties is a key requirement in ECG processing $[39,42,66]$.

In this study, the re-parameterization of the standard ICA model is converted into a 'component model' which is geometric in nature and free of indeterminacies existing in standard ICA (sICA). More importantly, this new perspective of ECG processing discussed suggests an extension to a more general model of multidimensional independent components $[9,15,52]$.

\section{Results}

In this study, case studies are carried out for ECG files taken from CSE database with and without parameterization. In these case studies, first the types of artefacts and noise are identified followed by their removal and obtaining the cleaned ECG signals using standard ICA. In this analysis, it is observed that the ECG signals and the uniformly distributed noise each define a one-dimensional source signal space, whereas the $\sin / \cos$ and the Gaussian noise-subspaces are two-dimensional. For the ECG data set, Jade algorithm is applied first to find smaller subspaces for MICA analysis and can therefore be regarded as a basis algorithm for parameterized MICA analysis.

Sources using Jade assumed non Gaussian distribution in this analysis, which in a way is known a priori only, but to fulfil some special requirements in ECG morphological studies, certain extensions of ICA have been studied and implemented on CSE based ECG data sets. However, if some weak prior information about the source ECG signals is available, even then the performance of the source separation algorithms may be significantly improved. 
In fact, one often has priors on the ECG signals, but may not be always necessary. A natural idea of adding the priors in the proposed ECG model was for simplifying or improving the standard ICA separation methods. The Jade algorithm is more appropriate for separating ECG data, because Jade yields lower matrix entries, i.e. higher reliability.

In this analysis, it is observed that the ECG signals and the uniformly distributed noise each define a one-dimensional source signal space, whereas the $\sin / \cos$ and the Gaussian noise-subspaces are two-dimensional. For the ECG data set, Jade algorithm is applied first to find smaller subspaces for MICA analysis and can therefore be regarded as a basis algorithm for parameterized MICA analysis. Using parameterization and MICA techniques the thresholds in this analysis is brought down to Kurtosis as 4.3 and for Varvar as 0.4, which indicates that the removal of noise and artefacts is faster in this study as compared to [42].

The simulation and graphical results are shown in the respective figures. The results have been obtained for number of points as 5000 and sampling frequency as $500 \mathrm{~Hz}$. Threshold criterions for kurtosis and variance of variance (Varvar) is obtained after testing 20-25, CSE database files as 4.3 and 0.4 after parameterization of ECG data which shows improvement in what is discussed in [42]. In the tables below, ICA1, ICA2 and ICA3 are the three extracted independent components. In this paper, 3-channel ECG data taken from CSE-database and each channel is $10 \mathrm{~s}$ duration, and sampling frequency of $500 \mathrm{~Hz}$ is used as reimplementation study and was first discussed in [42]. After applying ICA algorithm to the 3-channel ECG data, three independent components are obtained and then kurtosis of each independent component is calculated. A component whose modulus of kurtosis is below the threshold is marked as a noise component. Then each independent component is divided into 20 non-overlapping blocks, each of $0.5 \mathrm{~s}$ duration, as compared to 10 nonoverlapping blocks, each of $1 \mathrm{~s}$ duration as discussed in [42]. The variance of these 10 segments in a group of 2 for each component is calculated, and then the variance of these 10 variance values is obtained as the parameter Varvar. The component whose Varvar value is above the pre-determined threshold is marked as an artefact component [42]. In the graphs shown, ' $\mathrm{mV}$ ' refers to amplitude of an ECG waveform and $n=3$.

\subsection{Case studies}

CASE 1. Leads L1, L2 and L3 of File no-03 of CSE database for ECG are used in the simulation. This case deals with 3-channel ECG with baseline drift in the first two channels L1 and L2 and noise in the third channel L3.

From Tables 1 and 1(A), it is also observed that the value of kurtosis coefficients for ICA1 and ICA2 is increased, whereas ICA3, which represents the noise component in this case, is further reduced using PMICA technique. The Varvar coefficients of all three components ICA1, ICA2 and ICA3 are reduced using PMICA technique which shows its dominance over PsICA technique.

Table 1. The |Kurt| and Varvar values for each of the three ICA components for Case 1 for parameterized standard ICA (PsICA).

\begin{tabular}{llclc}
\hline Index & ICA1 & ICA2 & \multicolumn{1}{c}{ ICA3 } & Thresholds \\
\hline |Kurt| & 6.4660 & 29.6417 & $\mathbf{2 . 8 0 9 2}$ (Noise) & 4.3 \\
Varvar & 0.0186 & 0.1190 & 0.0098 & 0.4 \\
\hline
\end{tabular}

Thresholds for Kurtosis $=4.3$ and Varvar $=0.4$. 
Table 1A. The |Kurt| and Varvar values for each of the three ICA components for Case 1 for parameterized multidimensional ICA (PMICA).

\begin{tabular}{lcccc}
\hline Index & ICA1 & ICA2 & \multicolumn{1}{c}{ ICA3 } & Thresholds \\
\hline |Kurt| & 8.7860 & 32.6597 & $\mathbf{1 . 9 9 6 8 ( N o i s e )}$ & 4.3 \\
Varvar & 0.0113 & 0.1145 & 0.00088 & 0.4 \\
\hline
\end{tabular}

Thresholds for Kurtosis $=4.3$ and Varvar $=0.4$.

Analysis of this case indicates that channel L3 is a noise component, since it has $\mid$ Kurt $\mid<4.3$. It is apparent that channel-1 and channel-2 in Figure 4 has baseline wander and noise in the third channel L3 (Figure 5). It is obvious from Figure 6 and the Table 1, ICA3 is noise component since it has $\mid$ Kurt $\mid<4.3$, hence component ICA2 is made zero.

CASE 2. Leads AF, AL and AR of File no-04 of CSE database for ECG are used in the simulation. This case deals with 3-channel ECG with baseline drift in all three channels $\mathrm{AF}, \mathrm{AL}$ and AR. Channel AF has BLW as well as noise (Figures 7-10).

Analysis of this case indicates that channel, $\mathrm{AF}$ and $\mathrm{AL}$ are noise components, since they have $\mid$ Kurt $\mid<4.3$ (Table 2). It is obvious from Figure 11 and the Table 3, ICA1 and ICA2 are noise components since it has $\mid$ Kurt $\mid<4.3$, hence component ICA1 and ICA2 are made zero. ICA3 in this analysis appears to be undefined or in-deterministic (Figures 12-15).

The value of kurtosis coefficient for ICA3 obtained is a complex number, using PsICA algorithm and is $\mid$ Kurt| $[$ Kurtosis $(\mathbf{I C A 3})]=2.4599+0.0023 \mathrm{i}$ (Indeterminacy situation) .

The value of kurtosis coefficient for ICA3 obtained is also a complex number using PMICA algorithm and is |Kurt| [Kurtosis $(\mathbf{I C A 3})]=0.4647+0.0017 \mathrm{i}$ (Reduced Indeterminacy situation). From Tables 3 and 3(A), it is observed that the value of kurtosis coefficient for ICA3 obtained using PMICA algorithm is less than compared to the value of kurtosis coefficient for ICA3 obtained using PsICA algorithm and the indeterminacy is reduced in PMICA in comparison to PsICA case. ICA3 in this analysis thus still appears to be undefined or in-deterministic, but the indeterminacy content is reduced. Here still indeterminacy exists but the complex value of kurtosis coefficient for ICA3 is less in
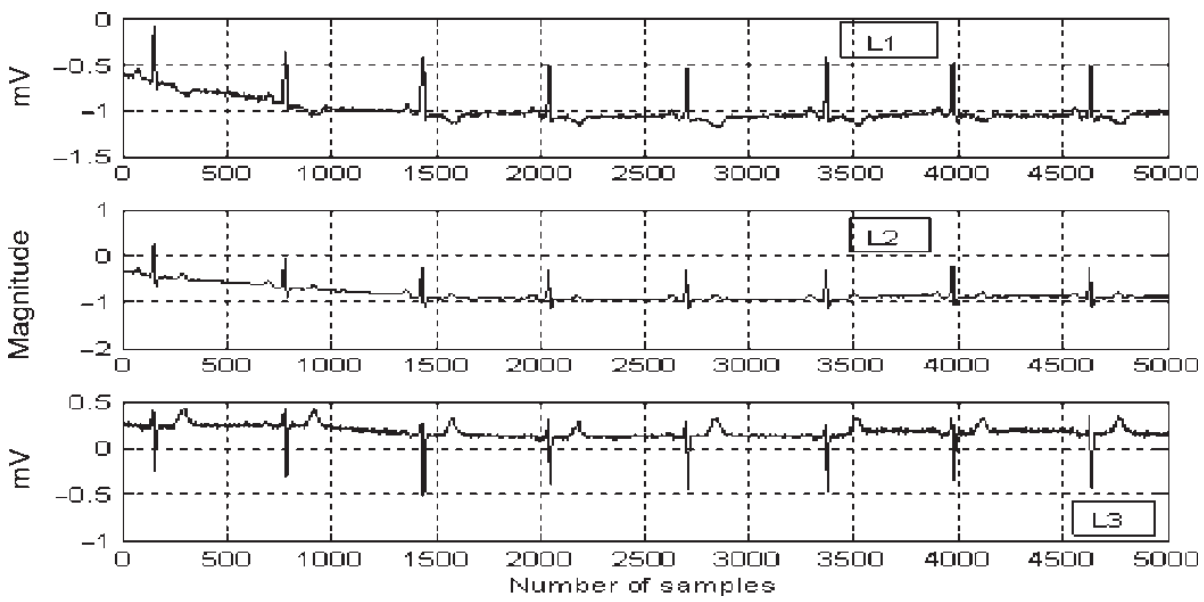

Figure 4. 3-Channel ECG with channels L1 and L2 having base line wander and noise in channel L3. 

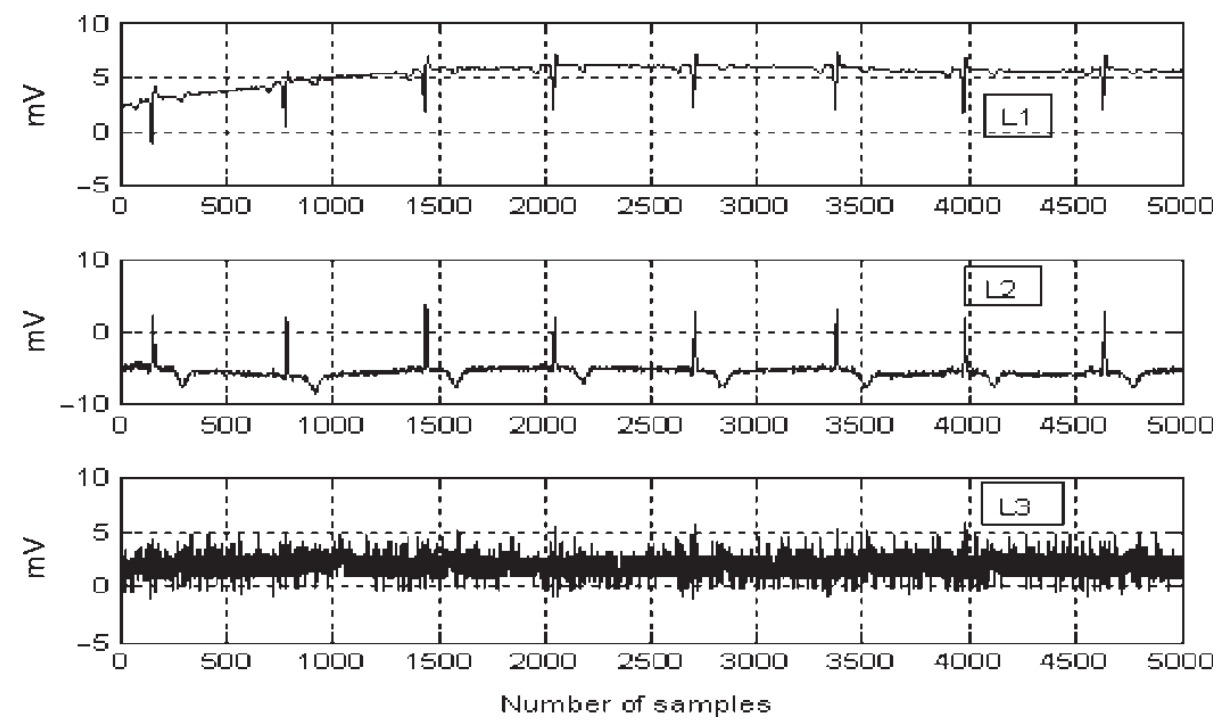

Figure 5. Extracted independent components.
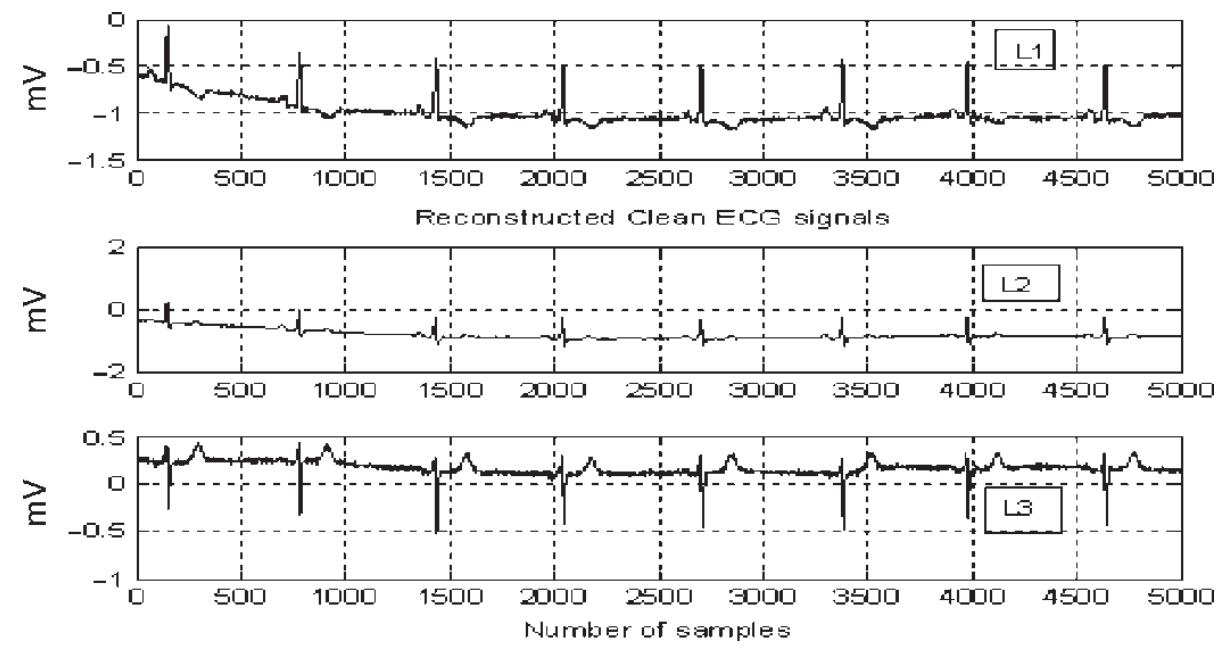

Figure 6. Reconstructed clean ECG signals.

PMICA as compared to PsICA. From Tables 3 and 3(A), it is also observed that the value of kurtosis coefficients for ICA1 and ICA2, which represents the noise component in this case, is further reduced using PMICA technique.

CASE 3. Leads V1, V2, V3 of a CSE base data file of ECG are used in the simulation. This case deals with 3-channel ECG having base-line wander in channel V1.

Analysis of this case indicates that channel V2 is a noise component, since it has $\mid$ Kurt $\mid<4$.3. It is apparent that channel-1 in Figure 16 has baseline wander (Figure 17). It is obvious from Figure 18 and that Table 1 ICA2 is noise component, since it has $\mid$ Kurt $\mid<4.3$, hence component ICA2 is made zero (Figures 19-21). 


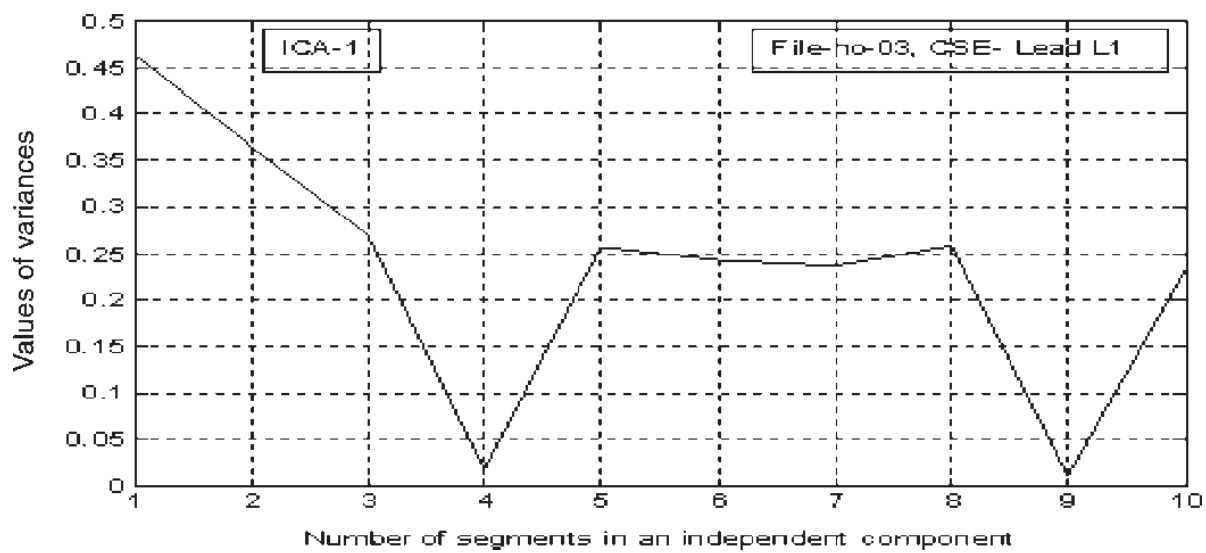

Figure 7. Plot of variance and number of segments in ICA1 (Lead L1).

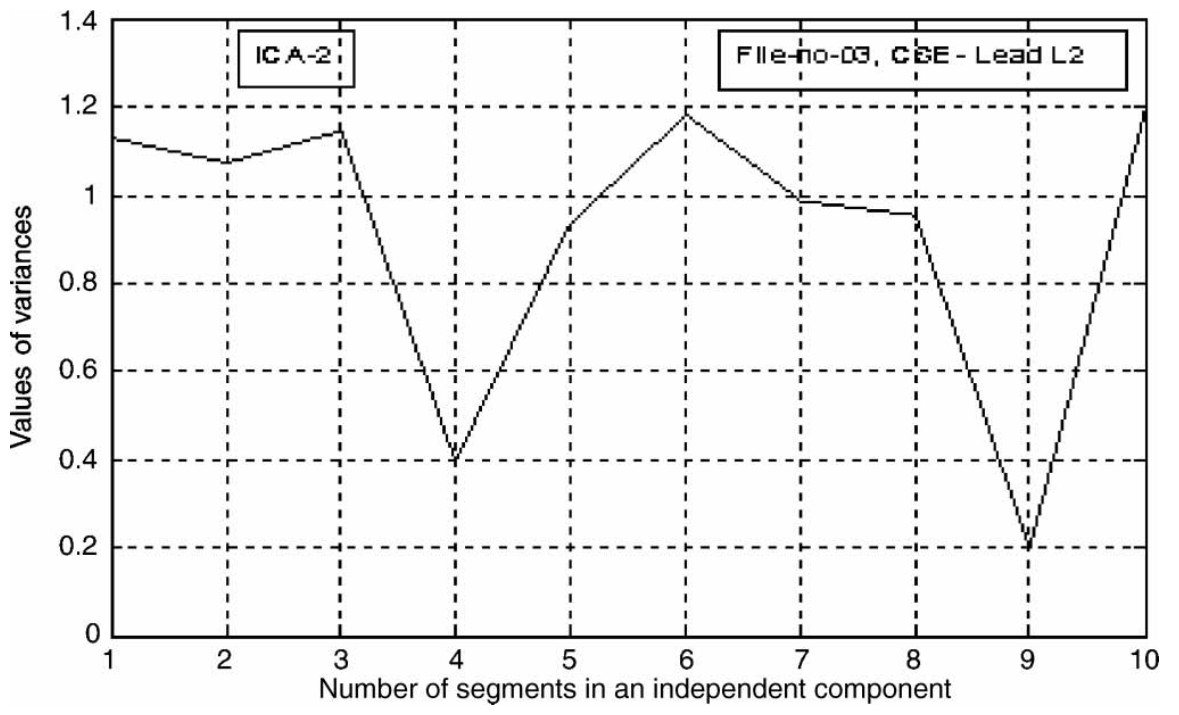

Figure 8. Plot of variance and number of segments in ICA2 (Lead L2).

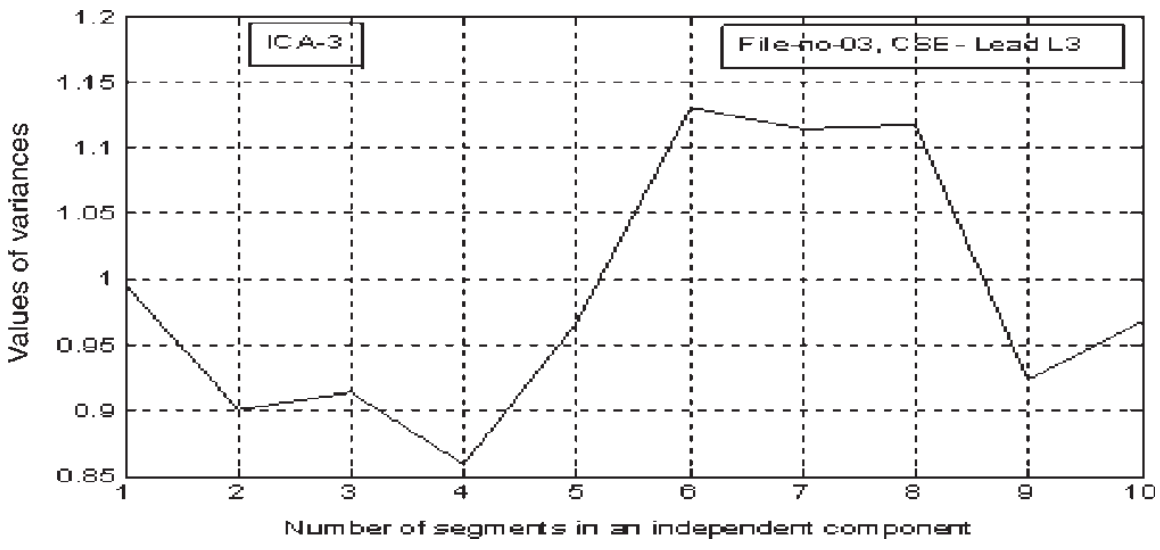

Figure 9. Plot of variance and number of segments in ICA3 (Lead L3). 

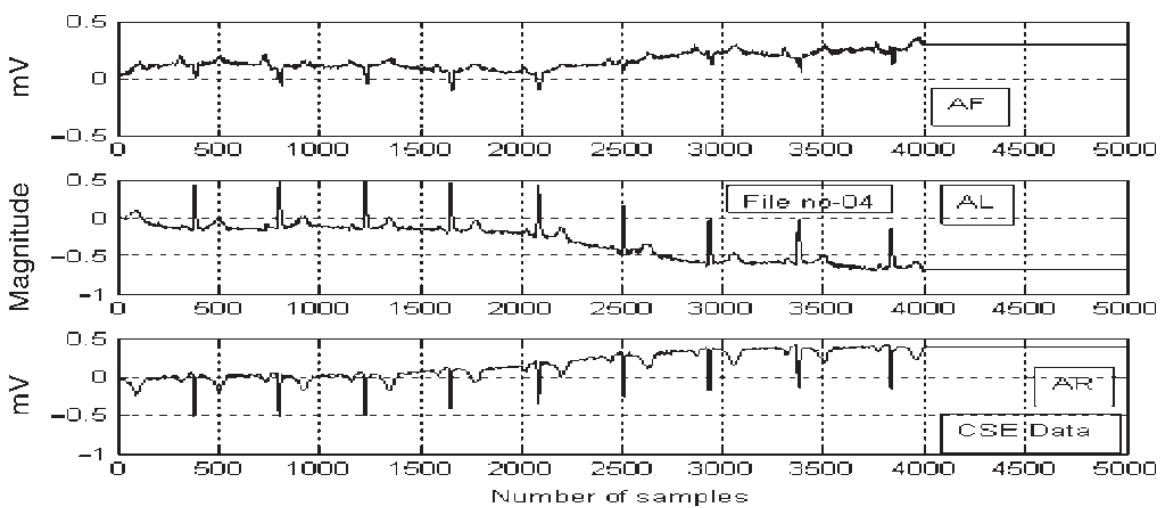

Figure 10. Three-channel ECG having base line wanders.

Table 2. Variance values of components ICA-1, ICA-2 and ICA-3 for PsICA.

\begin{tabular}{|c|c|c|c|c|}
\hline S. no & Segment number & $\begin{array}{c}\text { Comp } \\
\text { (ICA-1) L1 } \\
\text { (variances) }\end{array}$ & $\begin{array}{c}\text { Comp } \\
\text { (ICA-2) L2 } \\
\text { (variances) }\end{array}$ & $\begin{array}{c}\text { Comp } \\
\text { (ICA-3) L3 } \\
\text { (variances) }\end{array}$ \\
\hline 1 & Segment-1 & 0.4620 & 1.1304 & 0.9947 \\
\hline 2 & Segment-2 & 0.3653 & 1.0738 & 0.9003 \\
\hline 3 & Segment-3 & 0.2693 & 1.1475 & 0.9143 \\
\hline 4 & Segment- 4 & 0.0184 & 0.3968 & 0.8585 \\
\hline 5 & Segment-5 & 0.2563 & 0.9340 & 0.9666 \\
\hline 6 & Segment-6 & 0.2439 & 1.1855 & 1.1314 \\
\hline 7 & Segment-7 & 0.2370 & 0.9896 & 1.1150 \\
\hline 8 & Segment-8 & 0.2585 & 0.9540 & 1.1173 \\
\hline 9 & Segment-9 & 0.0111 & 0.1995 & 0.9233 \\
\hline 10 & Segment-10 & 0.2343 & 1.2057 & 0.9684 \\
\hline
\end{tabular}
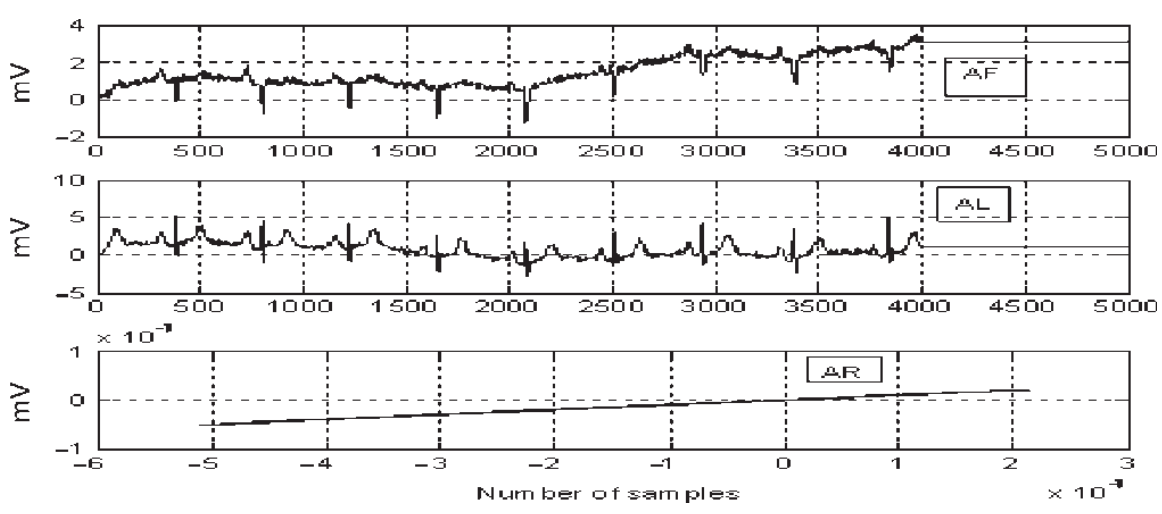

Figure 11. Extracted independent components for Case 2. 
Table 3. The |Kurt| and Varvar values for each of the three ICA components for Case 2 for parameterized standard ICA (PsICA).

\begin{tabular}{lllcc}
\hline Index & \multicolumn{1}{c}{ ICA1 } & \multicolumn{1}{c}{ ICA2 } & ICA3 & Thresholds \\
\hline |Kurt| & 1.8093 (Noise) & 4.1024 (Noise) & Complex Value & 4.3 \\
Varvar & 0.0073 & 0.1002 & 3.0284 -030 & 0.4 \\
\hline
\end{tabular}

Thresholds for Kurtosis $=4.3$ and Varvar $=0.4$.
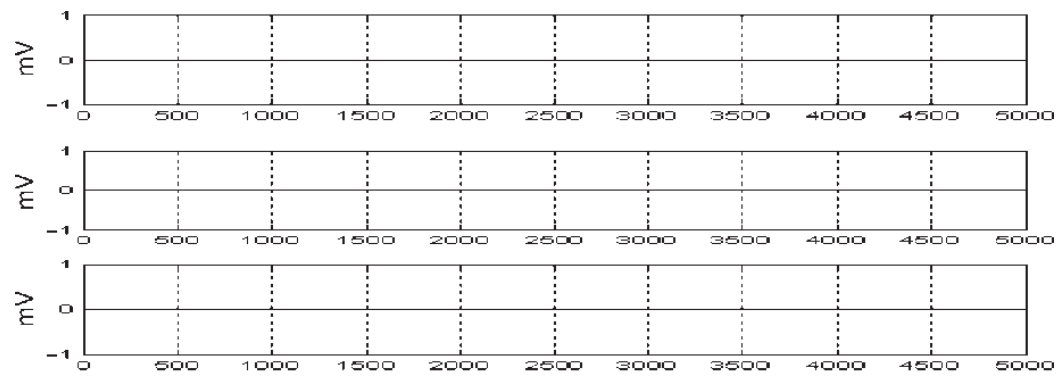

Figure 12. Reconstructed clean ECG signals for leads AF, AL and AR with loss of morphology.

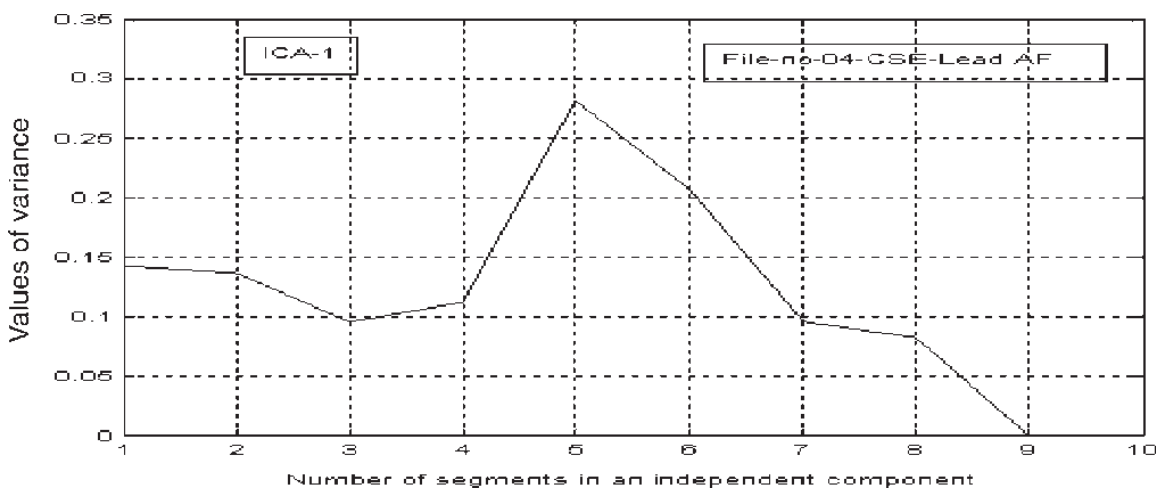

Figure 13. Plot of variance and number of segments in ICA1 (Lead AF).

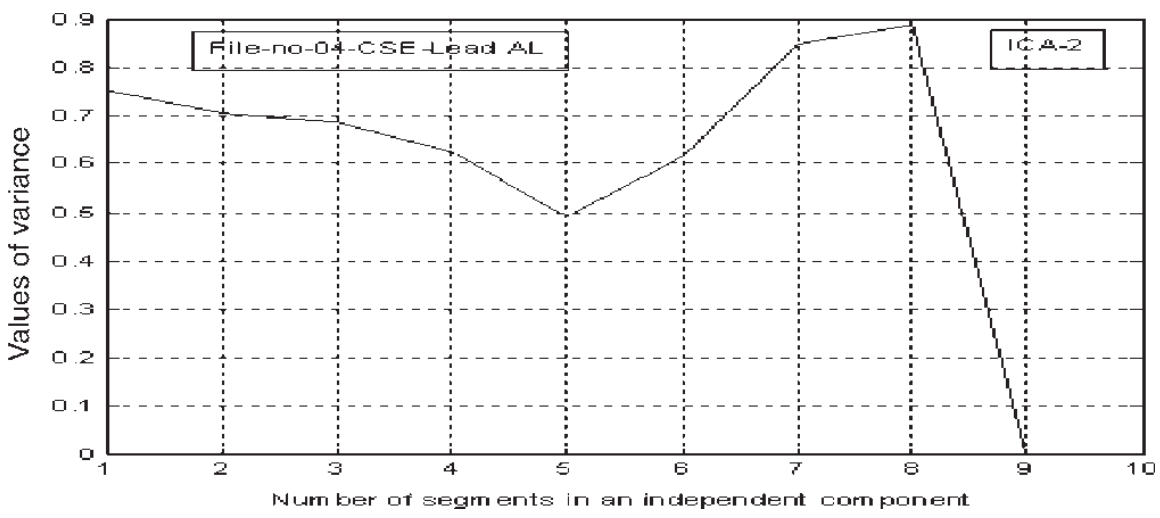

Figure 14. Plot of variance and number of segments in ICA2 (Lead AL). 
Table 3A. The |Kurt| and Varvar values for each of the three ICA components for Case 2 for parameterized multidimensional ICA (PMICA).

\begin{tabular}{llllc}
\hline Index & \multicolumn{1}{c}{ ICA1 } & \multicolumn{1}{c}{ ICA2 } & \multicolumn{1}{c}{ ICA3 } & Thresholds \\
\hline |Kurt| & 1.7645 (Noise) & 3.9457 (Noise) & $0.4647+0.0017 \mathrm{i}$ & 4.3 \\
Varvar & 0.0034 & 0.0987 & 0.0000 & 0.4 \\
\hline
\end{tabular}

Thresholds for Kurtosis $=4.3$ and Varvar $=0.4$.

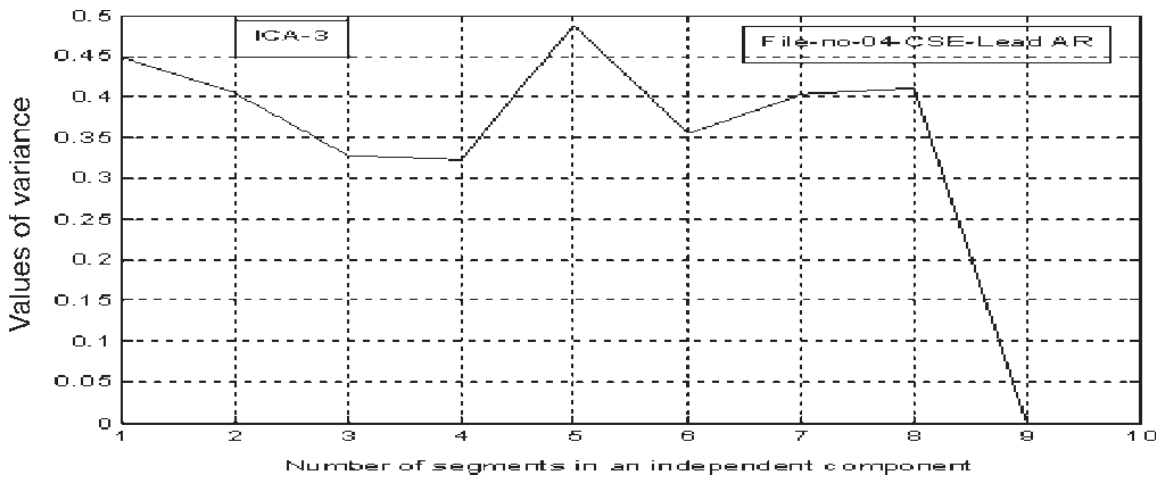

Figure 15. Plot of variance and number of segments in ICA3 (Lead AR).
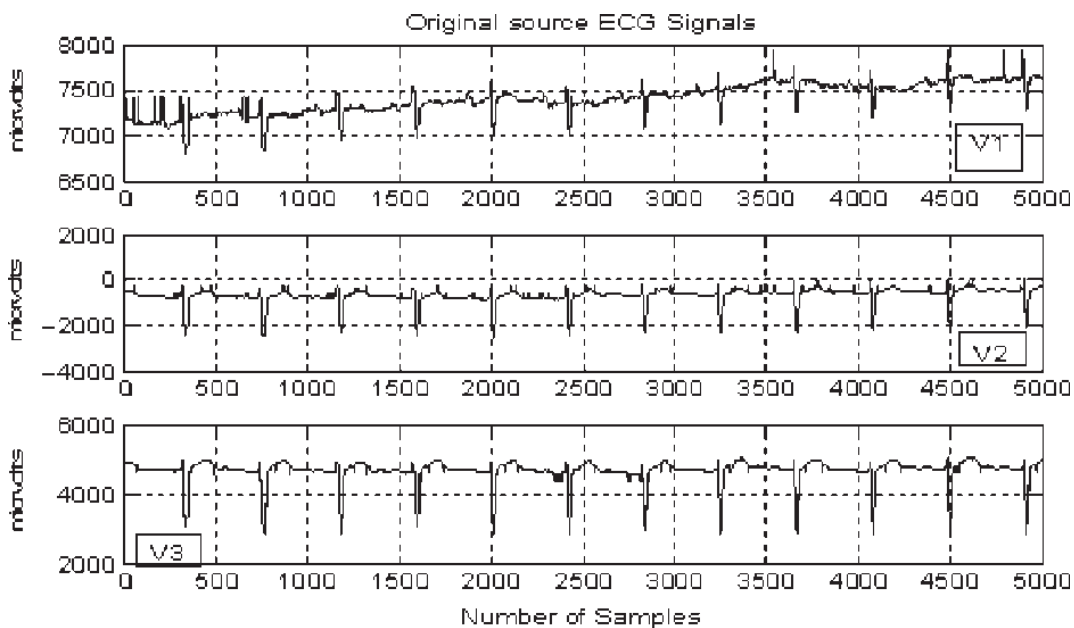

Figure 16. 3-Channel ECG, with channel V1 having base-line wander.

From Tables 4 and 4(A), it is also observed that the value of kurtosis coefficients for ICA1 and ICA3 is increased, whereas ICA2, which represents the noise component in this case, is further reduced using PMICA technique. The Varvar coefficients of all the three components ICA1, ICA2 and ICA3 are reduced using PMICA technique which in this case also shows its dominance over PsICA technique as discussed in Case 1 (Tables 5 and 6). 

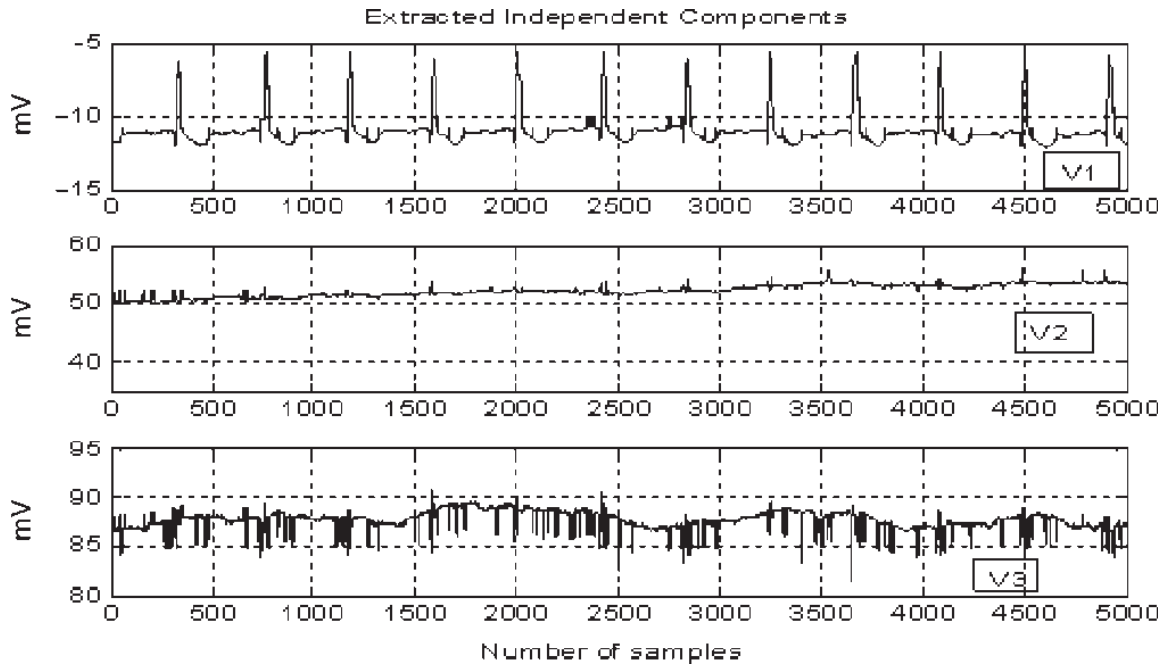

Figure 17. Extracted independent components.
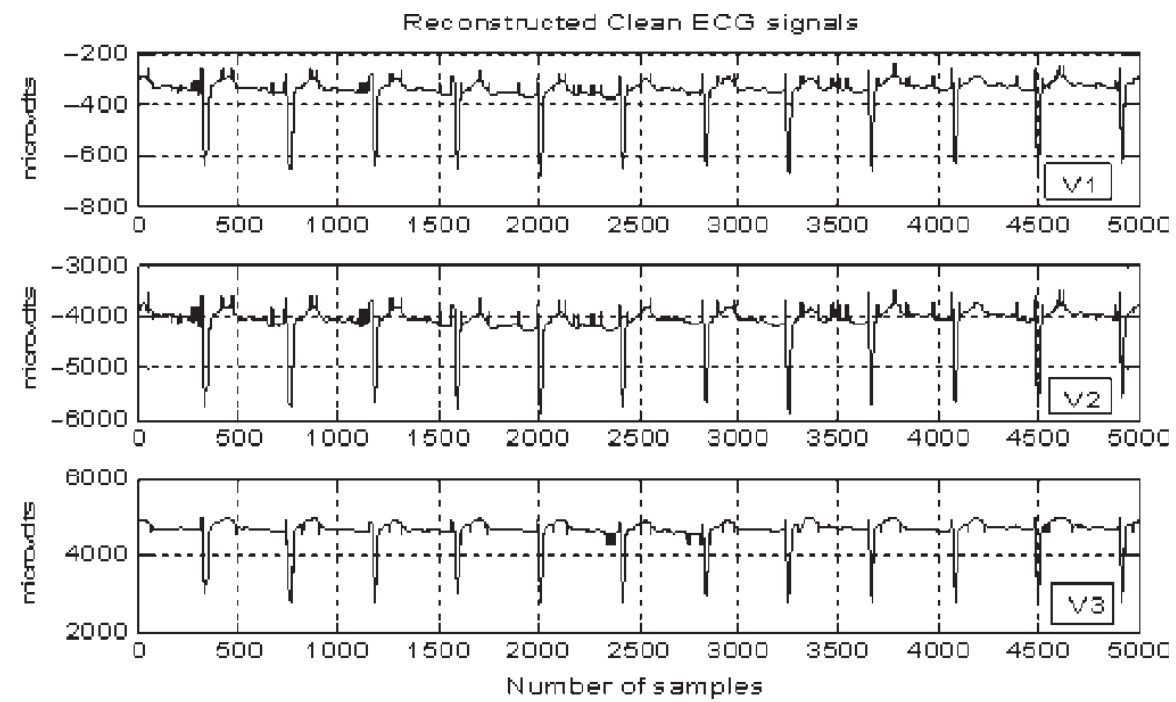

Figure 18. Reconstructed ECG signals for Case 3.

\section{Discussions}

ICA is a technique employed to solve a BSS problem that starts the research of the ECG sources from the hypothesis of statistical independence among them. In the basic or instantaneous ICA model, no time delay is involved in the mixing process and the signals picked up by the electrodes are a linear mixture of the sources. Hence, it would be at least reasonable to propose a modification or version of standard ICA technique to extract the important features and segments of ECG signals affected by artefacts and noise, thereby removing indeterminacies if available in the ECG data. By introducing the concept of irreducible independent subspaces or components, generalization to a parameter free mixture 


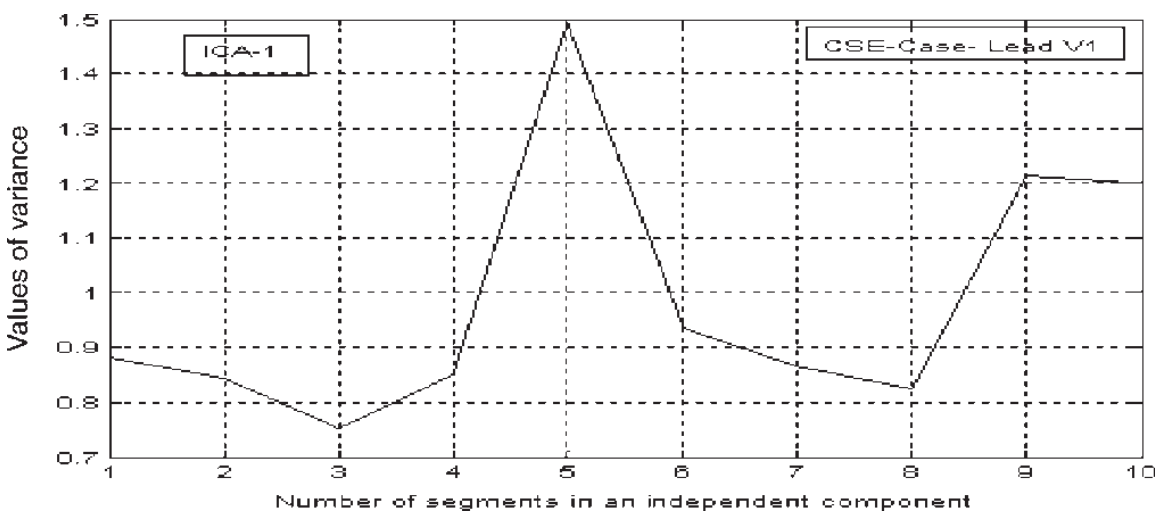

Figure 19. Plot of variance and number of segments in ICA1 (Lead V1).

Table 4. Values of |Kurt| and Varvar for each of the ICA components for Case 3 for parameterized standard ICA (PsICA).

\begin{tabular}{lrlr}
\hline Index & ICA1 & \multicolumn{1}{c}{ ICA2 } & ICA3 \\
\hline Kurt & 14.7901 & $\mathbf{2 . 2 0 2 0}$ (noise) & 5.0424 \\
Varvar & 0.0556 & 0.0037 & 0.0722 \\
\hline
\end{tabular}

Thresholds for Kurtosis $=4.3$ and Varvar $=0.4$.

of an ECG model can be presented. However, the condition of utmost one Gaussian can be relaxed by including previous results on non Gaussian component analysis of an ECG data set. After introduction of this general model, joint block diagonalization with unknown block sizes can be discussed and on the basis of a simple extension to Jade can be algorithmically performed to better understand the subspace analysis.

The indeterminacies if available in the ECG data was analysed using a modified version of Jade algorithm to PMICA and PsICA for comparative studies, as suggested in this study.

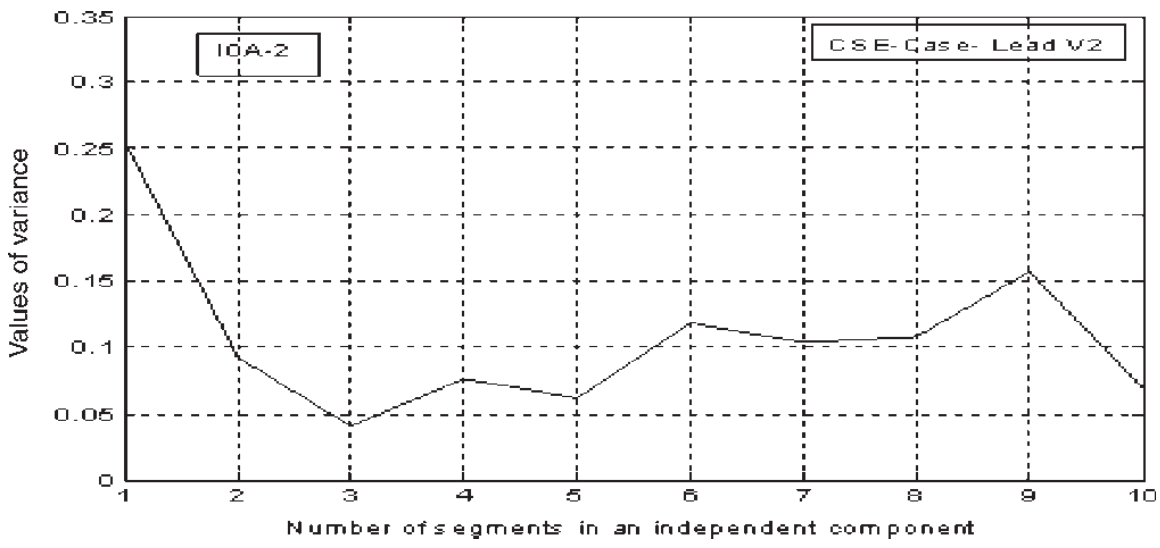

Figure 20. Plot of variance and number of segments in ICA2 (Lead V2). 


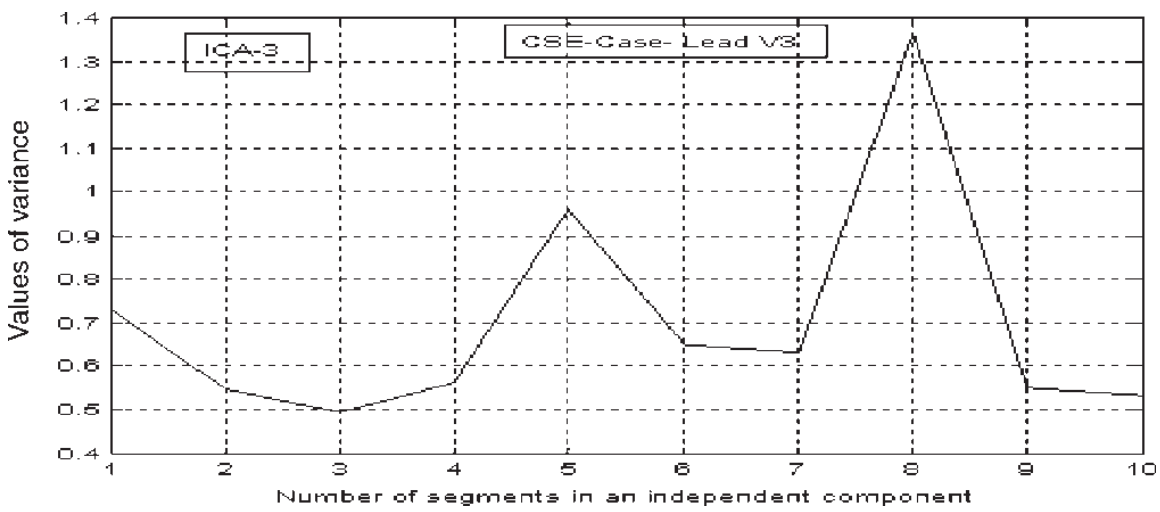

Figure 21. Plot of variance and number of segments in ICA3 (Lead V3).

Table 4A. The |Kurt| and Varvar values for each of the three ICA components for Case 3 for parameterized multidimensional ICA (PMICA).

\begin{tabular}{lclr}
\hline Index & ICA1 & \multicolumn{1}{c}{ ICA2 } & ICA3 \\
\hline |Kurt| & 15.6411 & $\mathbf{2 . 1 9 8 5}$ (noise) & 6.1426 \\
Varvar & 0.04856 & 0.00292 & 0.06988 \\
\hline
\end{tabular}

Thresholds for Kurtosis $=4.3$ and Varvar $=0.4$.

In this paper, the author has discussed a method for monitoring ECG signals by ICA approach, a special case of blind source separation. The ECG, noise and artefacts components are separated from multi-channel acquisitions by exploiting the well-known Jade algorithm available for instantaneous (standard) ICA model. By reconsidering the notion of ICA, a more general perspective can be envisioned: multidimensional independent component analysis. It is based on a geometric parameterization, which is free of the indeterminacies of matrix-based modelling. MICA relies on the idea of vectorvalued component rather than on scalar source signals. A canonical MICA decomposition is discussed as an invariant decomposition, which can be empirically computed by postprocessing the results of ICA decomposition. This calls for further research on developing

Table 5. Variance values of components ICA-1, ICA-2 and ICA-3 for Case 3.

\begin{tabular}{|c|c|c|c|c|}
\hline S. no & Segment number & $\begin{array}{c}\text { Comp } \\
\text { (ICA-1) V1 } \\
\text { (variances) }\end{array}$ & $\begin{array}{c}\text { Comp } \\
\text { (ICA-2) V2 } \\
\text { (variances) }\end{array}$ & $\begin{array}{c}\text { Comp } \\
\text { (ICA-3) V3 } \\
\text { (variances) }\end{array}$ \\
\hline 1 & Segment-1 & 0.8808 & 0.2542 & 0.7303 \\
\hline 2 & Segment-2 & 0.8435 & 0.0923 & 0.5485 \\
\hline 3 & Segment-3 & 0.7535 & 0.0405 & 0.4939 \\
\hline 4 & Segment-4 & 0.8516 & 0.0766 & 0.5624 \\
\hline 5 & Segment-5 & 1.4928 & 0.0617 & 0.9606 \\
\hline 6 & Segment-6 & 0.9358 & 0.1178 & 0.6491 \\
\hline 7 & Segment-7 & 0.8656 & 0.1043 & 0.6314 \\
\hline 8 & Segment- 8 & 0.8260 & 0.1084 & 1.3627 \\
\hline 9 & Segment-9 & 1.2138 & 0.1569 & 0.5509 \\
\hline 10 & Segment-10 & 1.1994 & 0.0682 & 0.5318 \\
\hline
\end{tabular}


Computational and Mathematical Methods in Medicine

Table 6. Variance values of components ICA-1, ICA-2 and ICA-3 for Case 2.

\begin{tabular}{lcccc}
\hline & & $\begin{array}{c}\text { Comp } \\
\text { (ICA-1) AF } \\
\text { (variances) }\end{array}$ & $\begin{array}{c}\text { Comp } \\
\text { (ICA-2) AL } \\
\text { (variances) }\end{array}$ & $\begin{array}{c}\text { Comp } \\
\text { (ICA-3) AR } \\
\text { (variances) }\end{array}$ \\
\hline 1 & Segment number & 0.1427 & 0.7511 & 0.4479 \\
2 & Segment-1 & 0.1365 & 0.7051 & 0.4041 \\
3 & Segment-2 & 0.0961 & 0.6865 & 0.3286 \\
4 & Segment-3 & 0.1116 & 0.6245 & 0.3221 \\
5 & Segment-4 & 0.2819 & 0.4921 & 0.4880 \\
6 & Segment-5 & 0.2066 & 0.6134 & 0.3552 \\
7 & Segment-6 & 0.0952 & 0.8470 & 0.4024 \\
8 & Segment-7 & 0.0822 & 0.8873 & 0.4103 \\
9 & Segment-8 & 0.0000 & 0.0000 & 0.0000 \\
10 & Segment-9 & 0.0000 & 0.0000 & 0.0000 \\
\hline
\end{tabular}

tools for detecting the existence of independent components in the ICA/MICA context. The important assumptions in applying ICA are: components are independent and have non Gaussian distributions. The ambiguities of ICA on the other hand are: energies of the extracted ICs cannot be determined and their order is undetermined. ICs of parameterized ECG signals displayed the desired aspects of the question at hand much more clearly than the ICs of the original ECG signals, while possibly also reduced the amount of ECG data to a fraction of the original ECG signal preserving morphology. In general, the usefulness of any blind decomposition method applied to ECG data is most likely relative to the fit between the assumptions of the algorithm. Therefore, it is important to consider the physiological basis of the delayed interactions between statistically defined independent components and the possible physiological significance of the derived components. Therefore, the author concludes that employing the time structure information in ICA calculations can potentially improve artefacts and noise removal and enhance the overall classification.

The salient advantages and contributions of the work proposed in this paper would benefit towards ECG analysis in various ways if used in practice, such as if the extracted independent components were further cleaned by statistical measures like kurtosis and Varvar (Variance of variance). In this study, it is also demonstrated that the value of kurtosis and Varvar coefficients for the independent components, which represents the noise and artefacts components can be further reduced using parameterized multidimensional ICA (PMICA) technique.

In addition to this, the indeterminacies if available in the ECG data are also analysed using a modified version of Jade algorithm developed for PMICA and parameterized standard ICA (PsICA) for comparative studies, highlighting merits and demerits. The indeterminacies if available in the ECG data are reduced in PMICA in a better way as compared to PsICA. The simulation results obtained indicate that ICA can definitely improve signal-noise ratio (SNR), like other higher order digital filtering methods such as Kalman, Butterworth etc. with minimum reconstruction errors. ICA of properly parameterized provided independent components (ICs) displayed more clearly the investigated properties of the original ECG sources. Lastly, in this analysis, it is also confirmed that, re-parameterization of the standard ICA model results into a component model using MICA technique, which is geometric in spirit and free of indeterminacies which generally exist in sICA model. 


\section{Conclusion}

The results of this analysis demonstrate that for ECG ICs, the threshold-based method was better in avoiding both overestimation as well as underestimation of ICs. As discussed in the simulation results, the combined method utilizing parameterization and MICA appears to be the best approach showing higher accuracy, automatization and better correction of electrocardiograms as well being good for feature classification also. The results for various CSE database files confirm that this approach can correctly identify the artefactual and noise ICs efficiently. However, the use of independent components raise a question of what their functional significance may be, that is, what is their functional relationship to the heart activities and ECG feature classification.

The method discussed provided a cleaning procedure for the noisy and corrupted ECG signals. Jade algorithm to the 3-channel ECG data is applied and it is observed that the approach is quite effective in identifying and removing noise/artefacts. The results obtained using Matlab environment demonstrate that there is significant improvement in signal quality i.e. SNR is improved. However, it was observed that sICA fails to separate the mixtures if more than one of the sources has a Gaussian amplitude distribution. It was felt by the author that it is important to determine how ICA algorithms can be designed to extract one-dimensional components and how they behave when processing of a mixture of multidimensional independent components is carried out. To describe the ECG signals more reliably for better extraction of diagnostic features, it is suggested that they can be modelled best with multidimensional components, which can be carried more rigorously as a future work.

\section{Acknowledgements}

The author is thankful to the Department of Electrical Engineering, Indian Institute of Technology, Roorkee, for providing the computing facilities to carry out this work. M.P.S. Chawla is grateful to G.S. Institute of Technology \& Science, Indore (M.P) and to AICTE, Govt. of India for sponsoring him for his doctoral research work.

\section{References}

[1] K. AbedMeraim, M.G. Amin, and A.M. Zoubir, Joint anti-diagonalization for blind source separation, Proc. ICASSP-01 2001.

[2] S. Achard and C. Jutten, Identifiability of post nonlinear mixtures, IEEE Signal Process. Lett. 12(5) (2005), pp. 423-426.

[3] L.B. Almeida, MISEP-linear and nonlinear ICA based on mutual information, Int. J. Mach. Learn. Res. 4 (2003), pp. 1297-1318.

[4] S.I. Amari, A. Cichocki, and H.H. Yang, A new learning algorithm for blind source separation, Adv. Neural inform. Process. Syst. 8 (1996), pp. 757-763.

[5] J. Anemuller, T. Sejnowski, and S. Makeig, Complex independent component analysis of frequency-domain EEG data, Neural Netw. 16 (2003), pp. 1313-1325.

[6] J. Anemuller, J.R. Duann, T.J. Sejnowski, and S. Makeig, Unraveling spatiotemporal dynamics in FMRI recordings using complex ICA, in Independent Component Analysis and Blind Signal Separation, C.G. Puntonet and A. Prieto eds., Springer-Verlag, Berlin, 2004, pp. 1103-1110.

[7] S. Araki, Sub-band based blind source separation for convolutive mixtures of speech, IEEE International Conference on Acoustics, Speech, and Signal Processing (2003), pp. 509-512.

[8] B. Azzerboni, G. Finocchio, M. Ipsale, F. La Foresta, and F.C. Morabito, A new approach to detection of muscle activation by independent component analysis and wavelet transform, Lecture Notes in Computer Science 2486, (2002), pp. 109-116.

[9] B. Azzerboni, M. Carpentieri, F. La Foresta, and F.C. Morabito, Neural-ICA and wavelet transform for artifacts Removal in surface EMG, in Proceedings of International Joint Conference on Neural Networks (IJCNN 2004), 2004, pp. 3223-3228. 
[10] F.R. Bach and M.I. Jordan, Finding clusters in independent component analysis, Proc. ICA2003 (2003), pp. 891-896.

[11] W. Baumann, B.U. Kohler, D. Kolossa, and R. Orglmeister, Real time separation of convolutive mixtures, in T.W. Lee, T.P. Jung, S. Makeig and T.J. Sejnowski eds., (2001), pp. 65-69, 3rd International Conference on Independent Component Analysis and Blind Signal Separation, San Diego, CA.

[12] B. Azzerboni, F. La Foresta, N. Mammone, and F. Carlo, A new approach based on waveletICA algorithms for fetal electrocardiogram extraction, ESANN-05, in Proceedings of the European Symposium on Artificial Neural Networks, Belgium, 2005, pp. 193-198.

[13] D. Callaerts, Signal separation based on singular value decomposition and their application to the real time extraction of the fetal electrocardiogram from cutaneous recordings, E.E. Dept, KU Leuven, 1989.

[14] G. Camps, M. Martinez, and E. Soria, Fetal ECG extraction using an FIR neural network, Comput. Cardiol. 28 (2001), pp. 249-252.

[15] J.F. Cardoso, Multidimensional independent component analysis, in IEEE, Proceedings of ICASSP '98, Seattle, WA 1998, pp. 1941-1944.

[16] — Blind signal separation: Statistical principles, Proc. IEEE 9 (1998), pp. 2009-2025.

[17] - The three easy routes to independent component analysis: contrasts and geometry, Proceedings of ICA-2001, San Diego, USA, 2001.

[18] J.F. Cardoso and B.H. Laheld, Equivariant adaptive source separation, IEEE Trans. Signal Process. 44 (1996), pp. 3017-3030.

[19] — An information-maximization approach to blind separation and blind deconvolution, IEEE Trans. Signal Process. 44 (1996), pp. 3017-3030.

[20] J.F. Cardoso and D.T. Pham, Optimization issues in noisy Gaussian ICA, in Independent Component Analysis and Blind Signal Separation, C.G. Puntonet and A. Prieto eds., SpringerVerlag, Berlin, 2004, pp. 41-48.

[21] F. Castells, J.J. Rieta, J. Millet, and V. Zarzoso, Spatiotemporal blind source separation approach to atrial activity estimation in atrial tachyarrhythmias, IEEE Trans. Biomed. Engg. 52 (2005), pp. 258-267.

[22] M.P.S. Chawla, Parameterization and correction of electrocardiogram signals using independent component analysis, WSPC, Singapore Int. J. Mech. Med Biol 7(4) (2007), pp. 355-379.

[23] — Parameterization and R-peak error estimations of ECG signals using independent component analysis, Taylor \& Francis Int. J. Comput. Math. Methods Med. 8(4) (2007), pp. $263-285$.

[24] - Segment classification of ECG data and construction of scatter plots using principal component analysis, Int. J. Mech. Med. Biol. (2008), In Press.

[25] M.P.S. Chawla, H.K. Verma, and V. Kumar, ECG modeling and QRS detection using principal component analysis, Proceedings of IET, Int. Conference, MEDSIP-06, Glasgow, UK, 2006.

[26] - Independent component analysis: A novel technique for removal of artifacts and baseline wander in ECG, in Proceedings of National Conference, CISCON-2006, MIT, Manipal, India, 2006, pp. 14-18.

[27] - Modeling and feature extraction of ECG using independent component analysis, IET Proceedings, Int. Conference, APSCOM-2006, Hong Kong, 2006.

[28] M.P.S. Chawla, H.K. Verma, and V. Kumar, A new statistical PCA-ICA algorithm for location of R-peaks in ECG, Elsevier Int. J. Cardiol (2007), In Press.

[29] - Artifacts and noise removal in electrocardiograms using independent component analysis, Int. J. Cardiol (2007), In Press.

[30] A. Cichocki and S. Amari, Adaptive Blind Signal and Image Processing, John Wiley and Sons, New York, 2002.

[31] A. Cichocki, R. Unbehauen, and E. Rummert, Robust learning algorithm for blind separation of signals, Electron. Lett. 30(17) (1994), pp. 1386-1387.

[32] P. Comon, Independent component analysis - a new concept?, Signal Process. 36 (1994), pp. 287-314.

[33] T. Cover and J. Thomas, Elements of Information Theory, Wiley Series in Telecommunications, John Wiley and Sons, New York, 1991.

[34] G.A. Darbellay and P. Tichavsky, Independent component analysis through direct estimation of the mutual information, ICA-2000, Helsinki, Finland (2000), pp. 69-74. 
[35] M. Davies and N. Mitianoudis, Audio source separation of convolutive mixtures, IEEE Trans. Speech Audio Process. 11(5) (2003), pp. 489-497.

[36] B. Draper, K. Baek, M.S. Bartlett, and J.R. Beveridge, Recognizing faces with PCA and ICA, Special Issue on Face Recognition Comput. Vis. Image Underst. 91(1-2) (2003), pp. 115-137.

[37] M. Dyrholm, Independent component analysis in a convoluted world, Kongens, Lyngby, Denmark, IMM158, 2005.

[38] M. Dyrholm, S. Makeig, and L.K. Hansen, Model structure selection in convolutive mixtures, in Independent Component Analysis And Blind Signal Separation, J. Rosca, D. Erdogmus, J.C. Principe and S. Haykin eds., Charleston, USA, 2006, pp. 74-81.

[39] - Model selection for convolutive ICA with an application to spatiotemporal analysis of EEG, Neural Comput. 19 (2007), pp. 934-955.

[40] M. Enescu, Adaptive methods for blind equalization and signal separation in MIMO systems, Dsc-Tech, Thesis, Helsinki Univ. Tech, Signal Processing Laboratory, 2002.

[41] P. Gao, E.C. Chang, and L. Wyse, Blind Separation of fetal ECG from single mixture using SVD and ICA, Proceedings of ICICS-PCM, Singapore, December (2003), p. 2003.

[42] T. He, G. Clifford, and L. Tarassenko, Application of independent component analysis in removing artefacts from the electrocardiogram, Springer-Verlag, London Neural Comput. Appl. (2005), pp. 1-19.

[43] J. Herault and C. Jutten, Space or time adaptive signal processing by neural networks models, Int. Conf. on Neural Networks for Computing, Snowbird, UT, USA (1986), pp. 206-211.

[44] A. Hyvarinen and P.O. Hoyer, Emergence of phase and shift invariant features by decomposition of natural images into independent feature subspaces, Neural Comput. 12(7) (2000), pp. 1705-1720.

[45] - Emergence of phase and shift invariant features by decomposition of natural images into independent feature subspaces, Neural Comput. 12(7) (2000), pp. 1705-1720.

[46] A. Hyvarinen and E. Oja, A fast fixed-point algorithm for independent component analysis, Neural Comput. 9(7) (1997), pp. 1483-1492.

[47] A. Hyvarinen, P.O. Hoyer, and M. Inki, Topographic independent component analysis, Neural Comput. 13(7) (2001), pp. 1525-1558.

[48] A. HyvParinen, P.O. Hoyer, and M. Inki, Topographic independent component analysis, Neural Comput. 13(7) (2001), pp. 1525-1558.

[49] J. Iriarte, E. Urrestarazu, M. Valencia, M.M. Alegre, V. Armando, and A. Julio, Independent component analysis as a tool to eliminate artifacts in EEG: A quantitative study, J. Clin. Neurophysiol. 20(4) (2003), pp. 249-257.

[50] A. Irimia and L.A. Bradshaw, Artifact reduction in magnetogastrography using fast independent component analysis, Physiol. Meas. 26 (2005), pp. 1059-1073.

[51] C.J. James and C.W. Hesse, Independent component analysis for biomedical signals, Physiol. Meas. 26 (2005), pp. R15-R39.

[52] C. Jutten and J. Herault, Blind separation of sources, Part I: An adaptive algorithm based on a neuromimetic architecture, Signal Process. 24(1) (1991), pp. 1-10.

[53] — Independent components analysis versus principal components analysis, Proc. European Signal Processing Conf. (EUSIPCO 88), Grenoble, France (1988), pp. 643-646.

[54] C. Jutten and J. Karhunen, Advances in blind source separation (BSS) and independent component analysis (ICA) for nonlinear mixtures, Int. J. Neural Syst. 14(5) (2004), pp. 1-26.

[55] C. Jutten and M.B. JuttenZadeh, Source separation: Principles, current advances and applications, Notes (1997), pp. 1-10.

[56] D. Knezevic, Blind source separation for signal processing applications, University of Western Australia (2004).

[57] V. Krishnaveni, S. Jayaraman, P.M.M. Kumar, K. Shivakumar, and K. Ramadoss, Comparison of independent component analysis algorithms for removal of ocular artifacts from electroencephalogram, Meas. Sci. Rev. J. 5(2) (2005), pp. 67-78.

[58] D.L. Lathauwer, B. De Moor, and J. Vandewalle, SVD-based methodologies for fetal electrocardiogram extraction, in Proceedings of the IEEE International Conference On Acoustic, Speech, Signal Processing, 2000, pp. 3771-3774.

[59] D.L. Lathauwer, B.D. Moor, and J. Vandewalle, Fetal electrocardiogram extraction by blind source subspace separation, IEEE Trans. Biomed. Engg. 47(5) (2000), pp. 567-572.

[60] J.K. Lin, Factorizing multivariate function classes, Adv. Neural Inform. Process. Syst. 10 (1998), pp. 563-569. 
[61] F. Meinecke, A. Ziehe, M. Kawanabe, and K.R. Muller, Estimating the reliability of ICA projections, in Advances in Neural Information Processing Systems. in S. Becker, T.G. Dietterich \& Z. Ghahramani eds., MIT Press, Cambridge, MA, 2002.

[62] S. Parmar and J. Sahambi, A comparative survey on removal of MECG artifacts from FECG using ICA algorithms, in Proceedings of International Conference on Intelligent Sensing and Information, 2004, pp. 88-91.

[63] L. Parra and C. Spence, Convolutive blind separation of non-stationary sources, IEEE Trans. Speech Audio Process. 8(3) (2000), pp. 320-327.

[64] D.T. Pham, Mutual information approach to blind separation of stationary sources, Proceedings of ICA-99, Aussois, France (1999), pp. 215-220.

[65] B. Poczos and A. Lorincz, Independent subspace analysis using k-nearest neighborhood distances, Proc. ICANN, vol. 3696 of LNCS, Warsaw, Poland, Springer (2005), pp. 163-168.

[66] F. Rong, L. Jose, and C. Vidal, Magnetoencephalographic artifact identification and automatic removal based on independent component analysis and categorization approaches, Int. J. Neurosci. Meth. (2006), pp. 337-354.

[67] F.G. Serrano, H.M. Bulla, and J.M. Fuentes, Independent component analysis applied to digital image watermarking, in IEEE International Conference on Acoustics, Speech and Signal Processing, 2001, pp. 1997-2000.

[68] L. Sornmo and P. Laguna, Bioelectrical signal processing in cardiac and neurological applications, Elsevier/Academic Press, London, UK, 2005.

[69] A. Taleb and C. Jutten, Source separation in post nonlinear mixtures, IEEE Trans. Signal Process. 47(10) (1999), pp. 2807-2820.

[70] J.M.A. Tanskanen, J.J. Viik, and J.A.K. Hyttinen, Independent component analysis of parameterized ECG signals, in Proceedings, 28th, IEEE, EMBS, Annual International Conference, New York City, USA, 2006, pp. 5704-5707.

[71] F.J. Theis, Uniqueness of complex and multidimensional independent component analysis, Signal Process. 84(5) (2004), pp. 951-956.

[72] - Uniqueness of real and complex linear independent component analysis revisited, Proc. of EUSIPCO, Vienna, Austria (2004), pp. 1705-1708.

[73] — Blind signal separation into groups of dependent signals using joint block diagonalization, IEEE Proc. (2005), pp. 5878-5881.

[74] — Towards a general independent subspace analysis, Notes, NIPS-06 (2006), pp. 1-8.

[75] - Multidimensional independent component analysis using characteristic functions, Notes, Institute of Biophysics, University of Regensburg, Germany, pp. 1-4.

[76] P. Tikkanen, Characterization and application of analysis methods for ECG and time interval variability data, University of Oulu, 1999.

[77] J. Urrestarazu, M. Iriarte, M. Alegre, C. Valencia, C. Viteri, and J. Artieda, Independent component analysis removing artifacts in ictal recordings, Epilepsia 45 (2004), pp. $1071-1078$

[78] V. Vigneron, A.P. Ionescu, A. Azancot, and O. Sibony, Fetal electrocardiogram extraction based on non-stationary ICA and wavelet denoising, Proceedings of ISSPA-2003 (2003).

[79] R. Vollgraf and K. Obermayer, Multi-dimensional ICA to separate correlated sources, Proc. of NIPS-2001 (2001), pp. 993-1000.

[80] F. Vrins, C. Jutten, and M. Verleysen, Sensors array and electrode selection for non- invasive fetal electrocardiogram extraction by independent component analysis, Proc. ICA-2004, LNCS, 3195 (2004), pp. 1017-1024.

[81] J.O. Wisbeck and R.O. Garcia, Application of neural networks to separate interferences and ECG signals, in Proceedings, IEEE International Caracas Conference on Devices, Circuits and Systems, 1998, pp. 291-294.

[82] M.B. Zadeh, On blind source separation in convolutive and nonlinear mixtures, INP, Grenoble, 2002.

[83] M.B. Zadeh and C. Jutten, A general approach for mutual information minimization and its application to blind source separation, Signal Process. 85(5) (2005), pp. 975-995. 


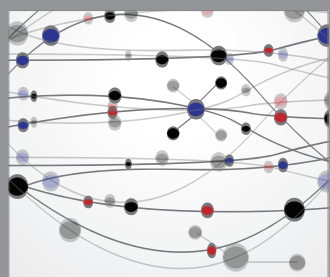

The Scientific World Journal
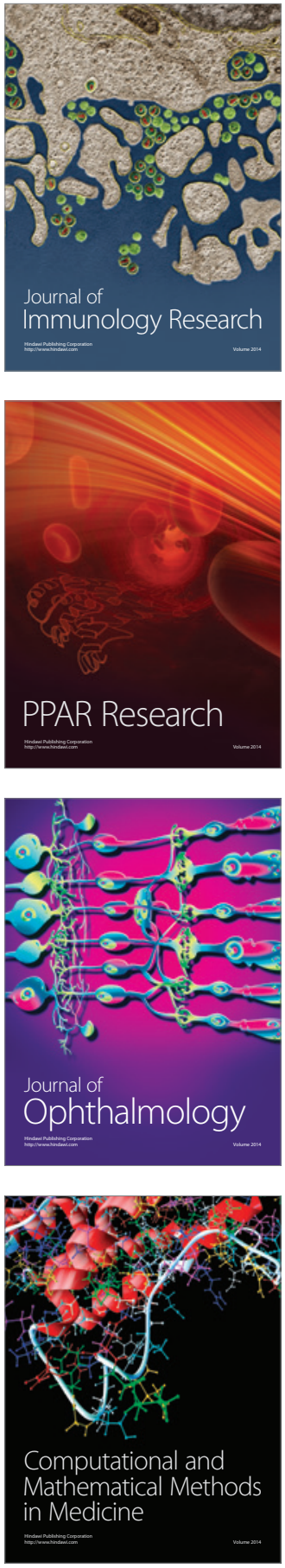

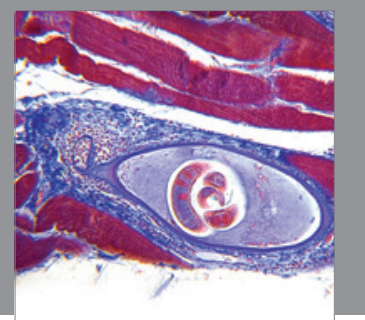

Gastroenterology

Research and Practice
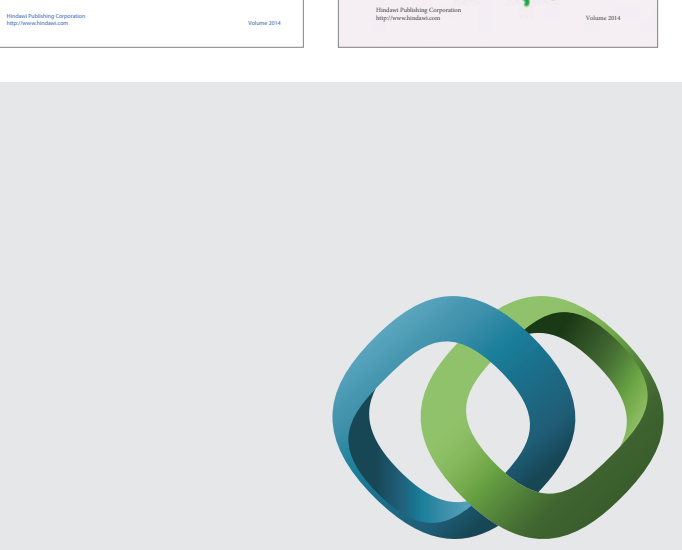

\section{Hindawi}

Submit your manuscripts at

http://www.hindawi.com
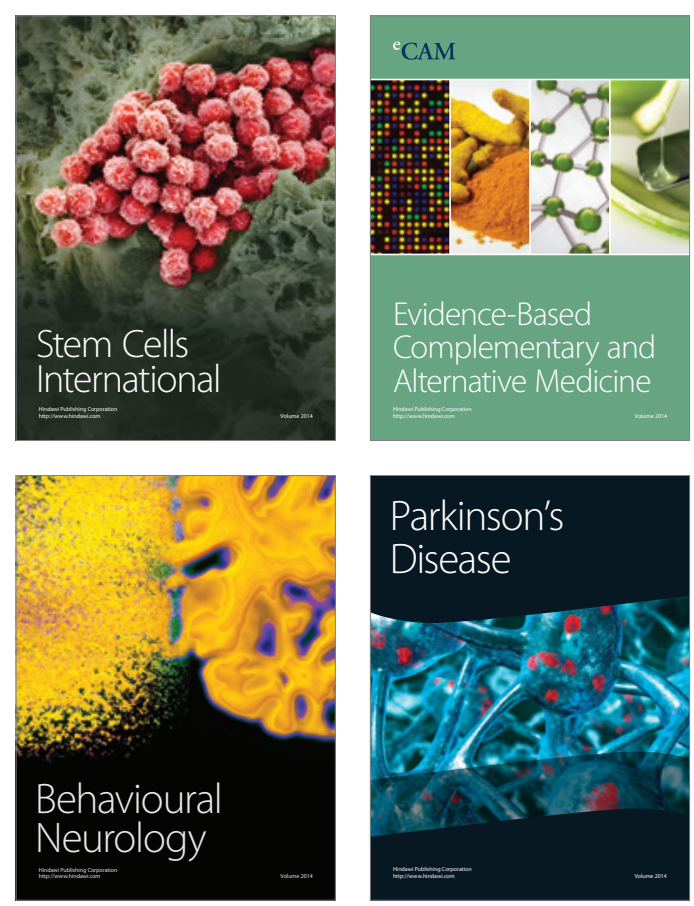

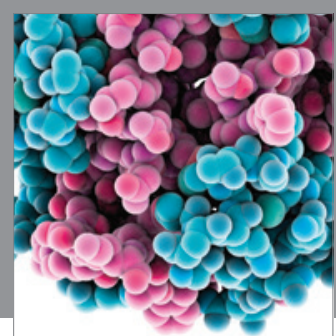

Journal of
Diabetes Research

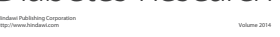

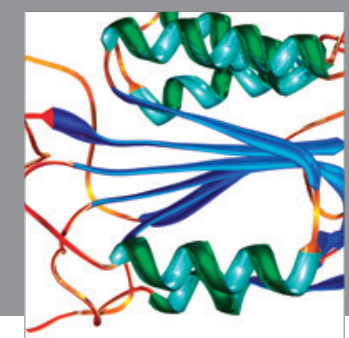

Disease Markers
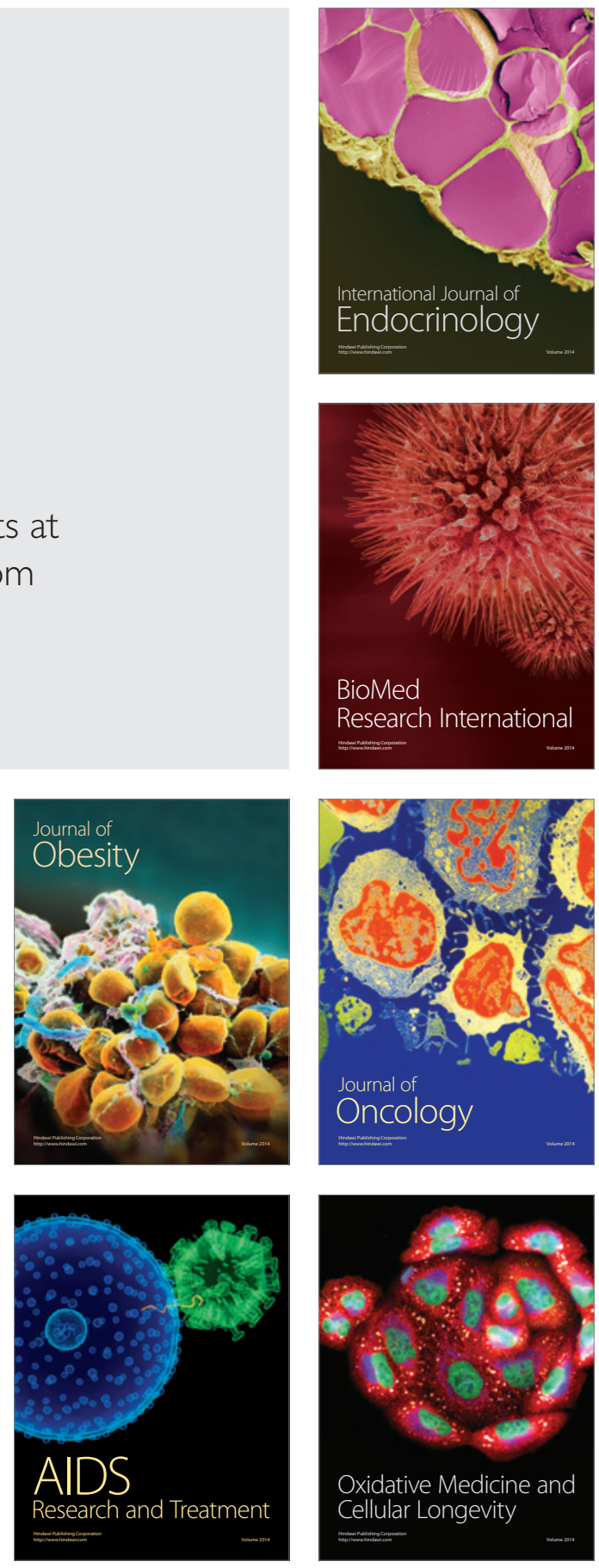